Article

\title{
Advancement of Non-Newtonian Fluid with Hybrid Nanoparticles in a Convective Channel and Prabhakar's Fractional Derivative-Analytical Solution
}

\author{
Muhammad Imran Asjad ${ }^{1}$, Noman Sarwar ${ }^{1}$, Muhammad Bilal Hafeez ${ }^{2}$ (D) Wojciech Sumelka ${ }^{2, *(D)}$ \\ and Taseer Muhammad ${ }^{3}$ \\ 1 Department of Mathematics, University of Management and Technology, Lahore 54770, Pakistan; \\ imran.asjad@umt.edu.pk (M.I.A.); nomansarwar243@gmail.com (N.S.) \\ 2 Institute of Structural Analysis, Poznan University of Technology, Piotrowo 5 Street, 60-965 Poznan, Poland; \\ Muhammad.hafeez@doctorate.put.poznan.pl \\ 3 Department of Mathematics, College of Sciences, King Khalid University, Abha 61413, Saudi Arabia; \\ tasgher@kku.edu.sa \\ * Correspondence: wojciech.sumelka@put.poznan.pl
}

check for

updates

Citation: Asjad, M.I.; Sarwar, N.; Hafeez, M.B.; Sumelka, W.;

Muhammad, T. Advancement of

Non-Newtonian Fluid with Hybrid

Nanoparticles in a Convective

Channel and Prabhakar's Fractiona Derivative-Analytical Solution.

Fractal Fract. 2021, 5, 99. https://

doi.org/10.3390/fractalfract5030099

Academic Editors: Burcu Gürbüz and Arran Fernandez

Received: 2 July 2021

Accepted: 14 August 2021

Published: 17 August 2021

Publisher's Note: MDPI stays neutral with regard to jurisdictional claims in published maps and institutional affiliations.

Copyright: (C) 2021 by the authors Licensee MDPI, Basel, Switzerland. This article is an open access article distributed under the terms and conditions of the Creative Commons Attribution (CC BY) license (https:// creativecommons.org/licenses/by/ $4.0 /)$

Abstract: The present paper deals with the advancement of non-Newtonian fluid containing some nanoparticles between two parallel plates. A novel fractional operator is used to model memory effects, and analytical solutions are obtained for temperature and velocity fields by the method of Laplace transform. Moreover, a parametric study is elaborated to show the impact of flow parameters and presented in graphical form. As a result, dual solutions are predicted for increasing values of fractional parameters for short and long times. Furthermore, by increasing nanoparticle concentration, the temperature can be raised along with decreasing velocity. A fractional approach can provide new insight for the analytical solutions which makes the interpretation of the results easier and enable the way of testing possible approximate solutions.

Keywords: non-Newtonian; fractional derivatives; vertical channel; nanoparticles; heat transfer

\section{Introduction}

Channel flow plays an important part in an inclusive range of industrial applications, such as heat exchangers in power plants and chemical reactors in the development industry. Although many processes of practical interest can be considered as two-phase flows by Newtonian behavior in both phases, there is a large number of related applications where the continuous liquid phase exhibits non-Newtonian flow features. Many examples can be found in the biochemical and biomedical as well as the food processing industries [1] Zheng et al. [2] investigated the impact of a vortex generator shape on liquids and the heat transformation of hybrid nanofluids in a channel. D'Ippolito et al. [3] studied the resistance of open channel flow because of vegetation at the research scale.

Nanofluids (term familiarized by Choi [4]) are considered identical in significant studies due to their thermal transfer development application. Some recent studies showed that the capability of heat transfer of nanofluids is more improved than of consistent fluids. Therefore, it is reasonable to exchange regular fluids with nanofluids. Because of their higher heat capability, the attention of numerous researchers is drawn toward the study of nanofluids. They play a dynamic role in industrial sectors such as electronics, transportation, foods, nuclear reactor, as well as biomedicine. The size of nanoparticles is very small, and these particles enhance the conductivity of base fluids when added to them. The construction of nanoparticles consists of metal oxide, carbon tubes, nitride, metal, and carbide [5]. Reddy et al. [6] addressed the performance of a micropolar thermal flux model magnesium oxide and molybdenum disulfide nanoparticle. The impact of a nanoparticle's mixture ratio, as well as the temperature and concentration on the thermal conductivity 
of hybrid nanofluids, was established by Wole-Osho et al. [7]. Haque et al. [8] discussed the laminar nanoparticles having heat transfer, using convection force in the presence of noncircular ducts. Khashi'ie et al. [9] deliberated a stream of hybrid nanoparticles due to melting heat transfer in the presence of a moving surface. Goldanlou et al. [10] introduced a heat exchanger pipe prepared by the edge-form of turbulators of the heat transfer of hybrid nanoparticles in an explosive. Montazer et al. [11] described the development of the original relationships and the heat transfer of the stormy nanoparticles. Benkhedda et al. [12] defined the horizontal tube considering the forms of nanoparticles, resulting in convective heat transfer presentation of the hybrid nanoparticles. Bhattad et al. [13] conferred the plate in the heat exchanger of hydrothermal presentation of dissimilar alumina hybrid nanoparticles. Izadi et al. [14] exclusively debated the three-sided cavity and the properties of porous solid on fleeting normal convection of the heat transfer of nanofluids.

Concerning the hybrid nanofluid, which is a combination of two diverse nanosized particles with base liquids, which influences the convective heat transfer, one can follow the following progress in the literature. Waini et al. [15] examined steady fully developed mixed convection flow of a lengthwise an upright surface, fixed in a porous medium with hybrid nanoparticles was examined. Pandya et al.'s [16] study focuses on the numerical model, developed to maximize the thermal performance of axial lined heat tube, working on $\mathrm{CeO}_{2}+\mathrm{MWCNT}$ / water-based hybrid nano-liquid. Babazadeh et al. [17] investigated the hybrid nano-liquid of a free convection transport inside a permeable average in the appearance of an outside applied magnetics force, modeling it through control volume, creating a fixed element technique. In Asadi et al.'s study [18], the discussion about the influence of hybrid nano-liquid $\mathrm{n}$ on the system performance was presented. Ikram et al. [19] studied clay nanoparticles for diverse-based liquids with a modern fractional scientific model that extracts nanofluids. Huminic et al.'s study [20] addressed dissimilar kinds of thermal structures aimed at dissimilar boundary situations and physical conditions, and contained a review of the contributions on entropy generation of the nano-liquid and hybrid nano-liquids. In their study, Nadeem et al. [21] addressed the base fluid ending in an exponentially stretching curved surface. The investigation evaluates the stimulus of including constraints of the hybrid nano-element.

Several fractional operators like the Grünwald-Letnikov derivative, Liouville and Riemann-Liouville derivatives, Caputo derivative, Marchaud derivative, Hadamard derivative, and Riesz derivative were applied as well [22,23]. Sene [24] studied the design of second-grade liquids, using the fractional differential equation through the Caputo Liouville fractional derivative. Mozafarifarda et al. [25] analyzed the fractional heat transfer equation for thin metal films utilizing the Caputo fractional derivative. Thabet et al. [26] studied the numerical findings of a novel disease of COVID-19 by using the mathematical method related to the existence and stability of $\mathrm{ABC}$.

In the recent literature, researchers have paid attention to the vertical geometry for fractional models with different operators but they ignored the fluid flow problem between two plates and obtained the fractional model through generalized constitutive laws with the Prabhakar fractional model. The main advantage of a fractional operator is only the kernel that consists of three parameters, but, namely, the generalized Mittag-Leffler function. The Prabhakar fractional operator with a fractional coefficient can be a useful tool for determining an appropriate mathematical model that can result in a good agreement between theoretical and real data. For a detailed discussion about the Prabhakar integral and its properties, see the following references [27-30]. Giusti and Colombaro [31] proposed a generalized fractional model for non-Newtonian fluid by using the generalized constitutes laws. The Prabhakar type fractional derivative contains a generalized Mittage-Leffler function as a kernel that is responsible for the history of fluid properties and controls the thermal and momentum boundary layers. Elnaqeeb et al. [32] studied an infinite vertical heated plate via Prabhakar-like thermal carriage. The generalized fractional constitutive equation aimed at shear stress proportion and thermal flux density vector of natural convective flows of Prabhakar-like fractional viscoelastic fluids via an infinite vertical 
frenzied wall was studied by Shah et al. [33]. Finally, in Akgül et al.'s study [34], the magnetohydrodynamics effects on heat transfer phenomena was examined. Wang et al. [35] discussed the Casson nanofluid with a modified Mittag-Leffler kernel of a Prabhakar form of unsteady thermal transportation flow.

In the presented state of the art, no work was applying a fractional operator in an important class of fluids, like non-Newtonian fluid between two vertical plates. The Prabhakar fractional derivative was used to model the channel flow problem, containing hybrid nanoparticles. Therefore, the prime interest is to find the analytical solutions of energy and momentum with the Laplace transform method and parametric analysis to show the flexibility of the proposed model.

\section{Materials and Methods}

Let us consider MHD convection flow through the microchannel of electrically conductive $\left(\mathrm{Ag}-\mathrm{TiO}_{2}-\mathrm{H}_{2} \mathrm{O}\right)$ hybrid nanofluid as shown in the Figure 1 under the following constraints:

(a) Microchannel length is infinite with width L;

(b) The channel is along $x$-axis and normal to $y$-axis;

(c) At $t \leq 0$, the temperature of the system is $T_{0}$;

(d) After $t=0^{+}$, the temperature increases from $T_{0}$ to $T_{w}$;

(e) Fluid accelerates in the $x$-direction;

(f) The magnetic field of strength $B_{0}$ works transversely to the flow direction.

The flow of electrically conductive $\mathrm{Ag}-\mathrm{TiO}_{2}-\mathrm{H}_{2} \mathrm{O}$ hybrid nanofluid causes the electromotive force, which yields current. Simultaneously, the induced magnetic field is ignored because of the hypothesis of a very small Reynolds number. Moreover, the electromagnetic force changes the intensity of the electric flux [36].

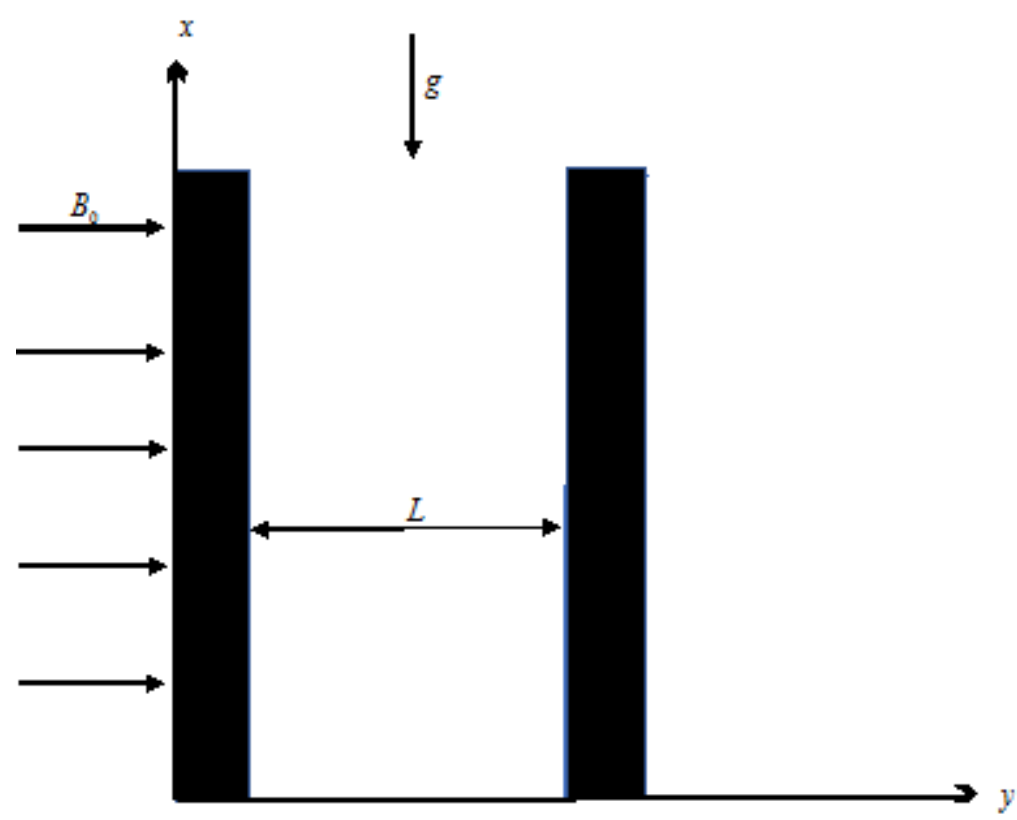

Figure 1. Flow geometry.

Within the above discussion, the governing equations for the stated problem are [37].

- The momentum equation

$$
\rho_{h n f}\left(\frac{\partial u(y, t)}{\partial t}+\beta_{b^{*}} u(y, t)\right)=\mu_{h n f} \frac{\partial^{2} u(y, t)}{\partial y^{2}}-\sigma_{h n f} B_{0}^{2} u(y, t)+g\left(\rho \beta_{T}\right)_{h n f}\left(T-T_{0}\right),
$$


- $\quad$ The energy balance equation

$$
\left(\rho C_{p}\right)_{h n f} \frac{\partial T(y, t)}{\partial t}=-\frac{\partial q(y, t)}{\partial y}
$$

- The generalized Fourier's law for thermal flux

$$
q(y, t)=-k_{h n f}{ }^{C} D_{\alpha, \beta, \alpha}^{\gamma} \frac{\partial T(y, t)}{\partial y} .
$$

We consider the following initial and boundary conditions:

$$
\begin{array}{ccc}
u(y, 0)=0, & T(y, 0)=T_{0}, & y \geq 0, \\
u(0, t)=0, & T(0, t)=T_{0} & t>0, \\
u(L, t)=0, & T(L, t)=T_{w}, & t>0,
\end{array}
$$

where the definition of the regularized Prabhakar derivative is $[32,38]$

$$
\begin{gathered}
{ }^{C} D_{\alpha, \beta, \alpha}^{\gamma} f(t)=E_{\alpha, m-\beta, \alpha}^{-\gamma} f^{(m)}(t)=e_{\alpha, m-\beta}^{-\gamma}(a ; t) * f^{(m)}(t)= \\
\int_{0}^{t}(t-\tau)^{m-\beta-1} E_{\alpha, m-\beta}^{-\gamma}\left(a(t-\tau)^{\alpha}\right) f^{(m)}(\tau) d \tau,
\end{gathered}
$$

where "*" represent the convolution product, $f^{(m)}$ denotes the mth derivative of $\mathrm{f}(\mathrm{t}) \in \mathrm{A}$

\begin{tabular}{|c|c|}
\hline Nanofluid & Hybrid Nanofluid \\
\hline$\rho_{n f}=(1-\phi) \rho_{f}+\phi \rho_{s}$ & $\rho_{h n f}=\left(1-\phi_{h n f}\right) \rho_{f}+\phi_{A g} \rho_{A g}+\phi_{\mathrm{TiO}_{2}} \rho_{\mathrm{TiO}_{2}}$ \\
\hline$\mu_{n f}=\frac{\mu_{f}}{(1-\phi)^{2.5}}$ & $\mu_{h n f}=\frac{\mu_{f}}{\left[1-\left(\phi_{A g}+\phi_{T i O_{2}}\right)\right]^{2.5}}$ \\
\hline$\left(\rho C_{p}\right)_{n f}=(1-\phi)\left(\rho C_{p}\right)_{f}+\phi\left(\rho C_{p}\right)_{s}$ & $\left(\rho C_{p}\right)_{h n f}=\left(1-\phi_{h n f}\right)\left(\rho C_{p}\right)_{f}+\phi_{A g}\left(\rho c_{p}\right)_{A g}+\phi_{T i O_{2}}\left(\rho C_{p}\right)_{T_{i O_{2}}}$ \\
\hline$\left(\rho B_{T}\right)_{n f}=(1-\phi)\left(\rho B_{T}\right)_{f}+\phi\left(\rho B_{T}\right)_{s}$ & $\left(\rho \beta_{T}\right)_{h n f}=\left(1-\phi_{h n f}\right)\left(\rho \beta_{T}\right)_{f}+\phi_{A g}\left(\rho \beta_{T}\right)_{A g}+\phi_{T i O_{2}}\left(\rho \beta_{T}\right)_{T i O_{2}}$ \\
\hline$\frac{\sigma_{n f}}{\sigma_{f}}=1+\frac{3\left(\frac{\sigma_{s}}{\sigma_{f}}-1\right) \phi}{\left(\frac{\sigma_{s}}{\sigma_{f}}+2\right)-\left(\frac{\sigma_{s}}{\sigma_{f}}-1\right) \phi}$ & 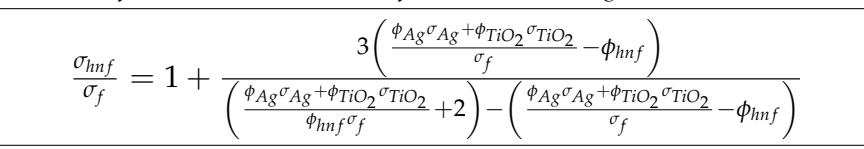 \\
\hline$\frac{K_{n f}}{K_{f}}=\frac{k_{s}+2 k_{f}-2 \phi\left(k_{s}-k_{f}\right)}{k_{s}+2 k_{f}+\phi\left(k_{s}-k_{f}\right)}$ & 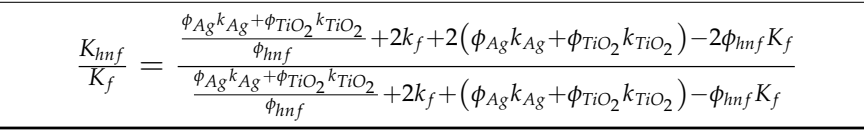 \\
\hline
\end{tabular}
$C^{m}(0, b)$ stands for the set of real-valued functions $f(t)$ whose derivatives are continuous up to order $(\mathrm{m}-1)$ on the interval $(0, \mathrm{~b})$ where $f^{(m-1)}(t)$ is absolutely continuous function, and $m=[\beta]$ represents the integer part of the parameter $\beta$.

For Equations (1)-(3), the thermophysical boundaries of the considered nanofluid and hybrid nanofluid are listed in Table 1.

Table 1. The thermophysical boundaries of the considered nanofluids.

where $\rho_{h n f}, \mathrm{u}(\mathrm{y}, \mathrm{t}), \beta_{b^{*}}, \mu_{h n f}, \sigma_{h n f}, B_{0}, \beta_{h n f},\left(C_{p}\right)_{h n f}, \mathrm{~T}(\mathrm{y}, \mathrm{t})$, and $k_{h n f}$ are the density, velocity, Brinkmann parameter, dynamic viscosity, electrical conductivity, magnetic field, thermal expansion, specific heat, temperature, and thermal conductivity, respectively.

Finally, for generality, we introduce the dimensionless form as

$$
\tau=\frac{v_{f}}{L^{2}} t, V=\frac{L}{v_{f}} u, Y=\frac{y}{L}, \theta=\frac{T-T_{0}}{T_{w}-T_{0}}, q^{*}=\frac{q}{q_{0}}, q_{0}=\frac{k_{h n f\left(T_{w}-T_{\infty}\right)} u_{0}}{v_{h n f}} .
$$


Therefore (neglecting the star notations), we have

$$
\begin{gathered}
A_{0}^{*}\left\{\frac{\partial V(Y, \tau)}{\partial \tau}+\beta_{b}^{*} V(Y, \tau)\right\}=A_{1}^{*} \frac{\partial^{2} V(Y, \tau)}{\partial Y^{2}}-A_{2}^{*} M V(Y, \tau)+A_{3}^{*} \operatorname{Gr} \theta(Y, \tau), \\
\operatorname{Pr} \frac{\partial \theta(Y, \tau)}{\partial \tau}=-\frac{\partial q(Y, \tau)}{\partial Y} \\
q(Y, \tau)=-{ }^{C} D_{\alpha, \beta, \alpha}^{\gamma} \frac{\partial \theta(Y, \tau)}{\partial Y}
\end{gathered}
$$

under the constraints

$$
\begin{array}{lll}
V(Y, 0)=0, & \theta(Y, 0)=0, & Y \geq 0, \\
V(0, \tau)=0, & \theta(0, \tau)=0, & \tau>0, \\
V(1, \tau)=0, & \theta(1, \tau)=1, & \tau>0,
\end{array}
$$

where

$$
\begin{gathered}
\beta_{b}^{*}=\frac{\beta_{b^{*}} L^{2}}{v_{f}}, M=\frac{L^{2} \sigma_{f} B_{0}^{2}}{\mu_{f}}, G r=\frac{g\left(\beta_{T}\right)_{f}\left(T_{w}-T_{0}\right) L^{3}}{v_{f}^{2}}, \operatorname{Pr}=\frac{\left(\mu C_{p}\right)_{f}}{k_{f}}, \lambda_{h n f}=\frac{k_{h n f}}{k_{f}}, A_{1}^{*}=\frac{1}{\left[1-\left(\Phi_{A g}+\Phi_{T i O_{2}}\right)\right]^{2.5}}, \\
A_{0}^{*}=1-\Phi_{h n f}+\frac{\Phi_{A g} \rho_{A g}+\Phi_{T i O_{2}} \rho_{T i O_{2}}}{\rho_{f}}, A_{2}^{*}=\frac{\sigma_{h n f}}{\sigma_{f}}, A_{3}^{*}=1-\Phi_{h n f}+\frac{\Phi_{A g}\left(\rho \beta_{T}\right)_{A g}+\Phi_{T i O_{2}}\left(\rho \beta_{T}\right)_{T i O_{2}}}{\left(\rho \beta_{T}\right)_{f}} .
\end{gathered}
$$

In the above equations, $\beta_{b}^{*}$ denotes Brinkman parameter, $\mathrm{M}$ is the magnetic field, $\mathrm{Pr}$ is the Prandtl number, and $G r$ is the Grashof number, respectively. It is essential that to accomplish the heat memory impacts, we additionally postulate the summed-up Fourier's law dependent on Prabhakar's fractional derivative, namely.

\section{Solution of the Problem}

\subsection{Temperature Field}

Applying the Laplace transform to Equations (10) and (11) with requirements $(13)_{2}$ and (14) ${ }_{2}$ and utilizing Prabhakar fractional derivative, we get for the temperature field

$$
\begin{gathered}
\operatorname{Pr} s \bar{\theta}(Y, s)=-\frac{\partial \bar{q}(Y, s)}{\partial y}, \\
\bar{q}(Y, s)=-s^{\beta}\left(1-a s^{-\alpha}\right)^{\gamma} \frac{\partial \bar{\theta}(Y, s)}{\partial Y} .
\end{gathered}
$$

In the following, using Equations (16) and (17), we have

$$
\frac{\partial^{2} \bar{\theta}(Y, s)}{\partial Y^{2}}-\frac{\operatorname{Pr} s \bar{\theta}(Y, s)}{s^{\beta}\left(1-a s^{-\alpha}\right)^{\gamma}}=0,
$$

which satisfies the following boundary conditions

$$
\bar{\theta}(0, s)=0, \quad \bar{\theta}(1, s)=\frac{1}{s}, \quad \tau>0 .
$$

The general solution of Equation (18) with conditions Equation (19) is

$$
\bar{\theta}(Y, s)=\frac{1}{s}\left[\frac{\sinh \left(Y \sqrt{\frac{P r s}{s^{\beta}\left(1-a s^{-\alpha}\right)^{\gamma}}}\right)}{\sinh \left(\sqrt{\frac{P r s}{s^{\beta}\left(1-a s^{-\alpha}\right)^{\gamma}}}\right)}\right] .
$$


It is important that Equation (20) can be written in the equivalent form

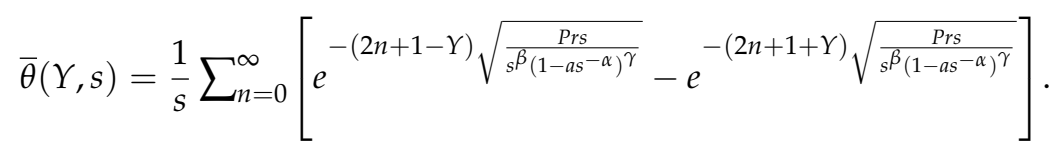

Moreover, Equation (21) can also be expressed in series form so that we can find the inverse of Laplace transform analytically, namely,

$$
\begin{aligned}
\bar{\theta}(Y, s)=\frac{1}{s}+[ & \left.\sum_{n=0}^{\infty} \sum_{m=1}^{\infty} \sum_{k=0}^{\infty} \frac{(-2 n-1+Y)^{m}(a)^{k}(P r)^{\frac{m}{2}} \Gamma\left(\frac{\gamma m}{2}+k\right)}{m ! k !} \frac{1\left(\frac{\gamma m}{2}\right)}{s^{\alpha k+\frac{\beta m}{2}-\frac{m}{2}+1}}\right]- \\
& {\left[\sum_{n=0}^{\infty} \sum_{l=0}^{\infty} \sum_{p=0}^{\infty} \frac{(-2 n-1-Y)^{l}(a)^{p}(\operatorname{Pr})^{\frac{l}{2}} \Gamma\left(\frac{\gamma l}{2}+p\right)}{l ! p !} \frac{1}{s^{\alpha p+\frac{\beta l}{2}}-\frac{l}{2}+1}\right] }
\end{aligned}
$$

with its inverse Laplace transform

$$
\begin{array}{r}
\theta(Y, \tau)=1+\sum_{n=0}^{\infty} \sum_{m=1}^{\infty} \sum_{k=0}^{\infty} \frac{\left.(-2 n-1+Y)^{m}(a)^{k}(P r)\right)^{\frac{m}{2}} \Gamma\left(\frac{\gamma m}{2}+k\right)}{m ! k ! \Gamma\left(\frac{\gamma m}{2}\right)} \frac{t^{\alpha k+\frac{\beta m}{2}-\frac{m}{2}}}{\Gamma\left(\alpha k+\frac{\beta m}{2}-\frac{m}{2}+1\right)}- \\
\sum_{n=0}^{\infty} \sum_{l=0}^{\infty} \sum_{p=0}^{\infty} \frac{(-(2 n+1+Y))^{l}(a)^{p}(P r)^{\frac{l}{2}} \Gamma\left(\frac{\gamma l}{2}+p\right)}{l ! p ! \Gamma\left(\frac{\gamma l}{2}\right)} \frac{t^{\alpha p+\frac{\beta l}{2}-\frac{l}{2}}}{\Gamma\left(\alpha p+\frac{\beta l}{2}-\frac{l}{2}+1\right)} .
\end{array}
$$

\subsection{Velocity Field}

By applying the Laplace transform to Equation (9) with constraints ,(12) ${ }_{1},(13)_{1}$ and $(14)_{1}$, we obtain

$$
\left[\frac{\partial^{2}}{\partial Y^{2}}-B_{1} s-B_{2}\right] \bar{V}(Y, s)=-B_{3} \bar{\theta}(Y, s),
$$

which satisfies the following conditions

$$
\bar{V}(0, s)=0, \quad \bar{V}(1, s)=0, \quad \tau>0 .
$$

The solution of Equation (24) subjected to constraint (25) is

$$
\begin{aligned}
& \bar{V}(Y, s)=-\frac{B_{4}}{s} \sum_{n=0}^{\infty}\left[\frac{e^{-(2 n)} \sqrt{\frac{P r s}{s^{\beta}\left(1-a s^{-\alpha}\right)^{\gamma}}}-e^{-(2 n+2)} \sqrt{\frac{P r s}{s^{\beta}\left(1-a s^{-\alpha}\right)^{\gamma}}}}{\left[1+\left(\frac{B_{1} s}{B_{2}}-\frac{P r s}{B_{2} s^{\beta}\left(1-a s^{-\alpha}\right)^{\gamma}}\right)\right]}\right] \times\left[\left(\sum_{m=0}^{\infty} e^{-(2 m+1-Y) \sqrt{\overline{B_{2+B}} B_{1}^{s}}}-\sum_{m=0}^{\infty} e^{-(2 m+1+Y) \sqrt{B_{2+B_{1} s}}}\right)\right]+ \\
& \frac{B_{4}}{s} \sum_{n=0}^{\infty}\left[\frac{e^{-(2 n+1-\gamma)} \sqrt{\frac{P r s}{s^{\beta}\left(1-a s^{-\alpha}\right)^{\gamma}}}-e^{-(2 n+1+\gamma)} \sqrt{\frac{P r s}{s^{\beta}\left(1-a s^{-\alpha}\right)^{\gamma}}}}{\left[1+\left(\frac{B_{1} s}{B_{2}}-\frac{P r s}{B_{2} s^{\beta}\left(1-a s^{-\alpha}\right)^{\gamma}}\right)\right]}\right],
\end{aligned}
$$

where

$$
B_{1}=\frac{A_{0}^{*}}{A_{1}^{*}}, \quad B_{2}=\frac{A_{2}^{*} M+\beta_{b}^{*}}{A_{1}^{*}}, \quad B_{3}=\frac{A_{3}^{*} G r}{A_{1}^{*}}, \quad B_{4}=\frac{B_{3}}{B_{2}} .
$$

Equation (26) can be written as

$$
\bar{V}(Y, s)=\overline{V_{1}}(Y, s)+\overline{V_{2}}(Y, s)+\overline{V_{3}}(Y, s)+\overline{V_{4}}(Y, s)+\overline{V_{5}}(Y, s)+\overline{V_{6}}(Y, s),
$$

where

$$
\overline{V_{1}}(Y, s)=-\frac{B_{4}}{s} \sum_{n=0}^{\infty}\left[\frac{e^{-(2 n) \sqrt{\frac{P r s}{s \beta\left(1-a s^{-\alpha}\right)^{\gamma}}}}}{\left[\left[1+\left(\frac{B_{1} s}{B_{2}}-\frac{P r s}{B_{2} s^{\beta}\left(1-a s^{-\alpha}\right)^{\gamma}}\right)\right]\right]}\right] \times \sum_{m=0}^{\infty}\left[e^{-(2 m+1-Y) \sqrt{B_{2}+B_{1} s}}\right],
$$




$$
\begin{aligned}
& \overline{V_{2}}(Y, s)=\frac{B_{4}}{s} \sum_{n=0}^{\infty}\left[\frac{e^{-(2 n) \sqrt{\frac{P r s}{s^{\beta}\left(1-a s^{-\alpha}\right)^{\gamma}}}}}{\left[1+\left(\frac{B_{1} s}{B_{2}}-\frac{P r s}{B_{2} s^{\beta}\left(1-a s^{-\alpha}\right)^{\gamma}}\right)\right]}\right] \times \sum_{m=0}^{\infty}\left[e^{-(2 m+1+Y) \sqrt{B_{2}+B_{1} s}}\right], \\
& \overline{V_{3}}(Y, s)=\frac{B_{4}}{s} \sum_{n=0}^{\infty}\left[\frac{e^{-(2 n+2) \sqrt{\frac{P r s}{s^{\beta}\left(1-a s^{-\alpha}\right)^{\gamma}}}}}{\left[\left[1+\left(\frac{B_{1} s}{B_{2}}-\frac{P r s}{B_{2} s^{\beta}\left(1-a s^{-\alpha}\right)^{\gamma}}\right)\right]\right]}\right] \times \sum_{m=0}^{\infty}\left[e^{-(2 m+1-Y) \sqrt{B_{2}+B_{1} s}}\right], \\
& \overline{V_{4}}(Y, s)=-\frac{B_{4}}{s} \sum_{n=0}^{\infty}\left[\frac{e^{-(2 n+2) \sqrt{\frac{P r s}{s^{\beta}\left(1-a s^{-\alpha}\right)^{\gamma}}}}}{\left[1+\left(\frac{B_{1} s}{B_{2}}-\frac{P r s}{B_{2} s^{\beta}\left(1-a s^{-\alpha}\right)^{\gamma}}\right)\right]}\right] \times \sum_{m=0}^{\infty}\left[e^{-(2 m+1+Y) \sqrt{B_{2}+B_{1} s}}\right] \text {, } \\
& \overline{V_{5}}(Y, s)=\frac{B_{4}}{s} \sum_{n=0}^{\infty}\left[\frac{e^{-(2 n+1-Y) \sqrt{\frac{P r s}{s^{\beta}\left(1-a s^{-\alpha}\right)^{\gamma}}}}}{\left[1+\left(\frac{B_{1} s}{B_{2}}-\frac{P r s}{B_{2} s^{\beta}\left(1-a s^{-\alpha}\right)^{\gamma}}\right)\right]}\right] \\
& \overline{V_{6}}(Y, s)=-\frac{B_{4}}{s} \sum_{n=0}^{\infty}\left[\frac{e^{-(2 n+1+Y) \sqrt{\frac{P r s}{s^{\beta}\left(1-a s^{-\alpha}\right)^{\gamma}}}}}{\left[\left[1+\left(\frac{B_{1} s}{B_{2}}-\frac{P r s}{B_{2} s^{\beta}\left(1-a s^{-\alpha}\right)^{\gamma}}\right)\right]\right]}\right] \text {. }
\end{aligned}
$$

It is challenging to find the inverse of Laplace transform of Equations (28)-(33), so we can rewrite it in a suitable series form.

$$
\begin{aligned}
& \overline{V_{1}}(Y, s)=B_{4} \sum_{n=0}^{\infty} \sum_{m=0}^{\infty} \sum_{\delta_{1}=0}^{\infty} \sum_{\delta_{2}=0}^{\infty} \sum_{\omega_{1}=0}^{\infty} \sum_{\omega_{2}=0}^{\infty} \sum_{z_{1}=0}^{\infty} \sum_{z_{2}=0}^{\infty} \sum_{z_{3}=0}^{\infty} \frac{(Y-2 m-1)^{\omega_{1}}\left(-B_{1}\right)^{z_{1}}(P r) \frac{\delta}{1}^{\frac{\delta_{1}}{2}}}{\delta_{1} \delta_{2} ! \omega_{1} ! \omega_{2} ! z_{2} ! z_{3} !\left(B_{1}\right)^{z_{2}-\omega_{2}}\left(B_{2}\right)^{\omega_{2}+z_{1}-\frac{\omega_{1}}{2}} \times} \\
& \frac{(-2 n)^{\delta_{1}}(-P r)^{z_{2}}(a)^{\delta_{2}+z_{3}}}{s^{\alpha \delta_{2}+\frac{\beta \delta_{1}}{2}-\frac{\delta_{1}}{2}-\omega_{2}+\beta z_{2}+\alpha z_{3}-z_{1}+1}} \times \frac{\Gamma\left(\frac{\gamma \delta_{1}}{2}+\delta_{2}\right) \Gamma\left(\frac{\omega_{1}}{2}+1\right) \Gamma\left(z_{1}+1\right) \Gamma\left(\gamma z_{2}+z_{3}\right)}{\Gamma\left(\frac{\gamma \delta_{1}}{2}\right) \Gamma\left(\frac{\omega_{1}}{2}+1-\omega_{2}\right) \Gamma\left(z_{1}+1-z_{2}\right) \Gamma\left(\gamma z_{2}\right)}, \\
& \overline{V_{2}}(Y, s)=B_{4} \sum_{n=0}^{\infty} \sum_{m=0}^{\infty} \sum_{\delta_{1}=0}^{\infty} \sum_{\delta_{2}=0}^{\infty} \sum_{q_{1}=0}^{\infty} \sum_{q_{2}=0}^{\infty} \sum_{z_{1}=0}^{\infty} \sum_{z_{2}=0}^{\infty} \sum_{z_{3}=0}^{\infty} \frac{(-(Y+2 m+1))^{q_{1}}\left(-B_{1}\right)^{z_{1}}(P r)^{\frac{\delta_{1}}{2}}}{\delta_{1 !} \delta_{2} ! q_{1} ! q_{2} ! z_{2} ! z_{3} !\left(B_{1}\right)^{z_{2}-q_{2}}\left(B_{2}\right)^{q_{2}+z_{1}-\frac{q_{1}}{2}}} \times
\end{aligned}
$$

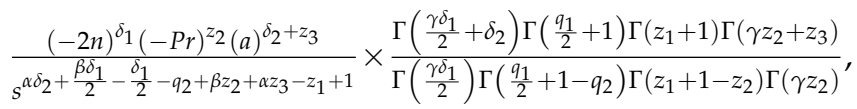

$$
\begin{aligned}
& \overline{V_{3}}(Y, s)=B_{4} \sum_{n=0}^{\infty} \sum_{m=0}^{\infty} \sum_{j_{1}=0}^{\infty} \sum_{j_{2}=0}^{\infty} \sum_{\omega_{1}=0}^{\infty} \sum_{\omega_{2}=0}^{\infty} \sum_{z_{1}=0}^{\infty} \sum_{z_{2}=0}^{\infty} \sum_{z_{3}=0}^{\infty} \frac{(Y-2 m-1)^{\omega_{1}}\left(-B_{1}\right)^{z_{1}}(P r)^{\frac{j_{1}}{2}}}{j_{1} ! j_{2} ! \omega_{1} ! \omega_{2} ! z_{2} ! z_{3} !\left(B_{1}\right)^{z_{2}-\omega_{2}}\left(B_{2}\right)^{\omega_{2}+z_{1}-\frac{\omega_{1}}{2}} \times} \\
& \frac{\left.(-2 n-2)^{j_{1}}(-P r)^{z}(a)\right)^{j_{2}+z_{3}}}{s^{\alpha j_{2}+\frac{\beta j_{1}}{2}-\frac{j_{1}}{2}-\omega_{2}+\beta z_{2}+\alpha z_{3}-z_{1}+1}} \times \frac{\Gamma\left(\frac{\gamma j_{1}}{2}+j_{2}\right) \Gamma\left(\frac{\omega_{1}}{2}+1\right) \Gamma\left(z_{1}+1\right) \Gamma\left(\gamma z_{2}+z_{3}\right)}{\Gamma\left(\frac{\gamma j_{1}}{2}\right) \Gamma\left(\frac{\omega_{1}}{2}+1-\omega_{2}\right) \Gamma\left(z_{1}+1-z_{2}\right) \Gamma\left(\gamma z_{2}\right)}, \\
& \overline{V_{4}}(Y, s)=-B_{4} \sum_{n=0}^{\infty} \sum_{m=0}^{\infty} \sum_{j_{1}=0}^{\infty} \sum_{j_{2}=0}^{\infty} \sum_{q_{1}=0}^{\infty} \sum_{q_{2}=0}^{\infty} \sum_{z_{1}=0}^{\infty} \sum_{z_{2}=0}^{\infty} \sum_{z_{3}=0}^{\infty} \frac{(-(Y+2 m+1))^{q_{1}}\left(-B_{1}\right)^{z_{1}}(P r)^{\frac{j_{1}}{2}}}{j_{1} ! j_{2} ! q_{1} ! q_{2} ! z_{2} ! z_{3} !\left(B_{1}\right)^{z_{2}-q_{2}}\left(B_{2}\right)^{q_{2}+z_{1}-\frac{q_{1}}{2}} \times} \\
& \frac{(-2 n-2)^{j_{1}}(-P r)^{z_{2}}(a)^{j_{2}+z_{3}}}{s^{\alpha j_{2}+\frac{\beta j_{1}}{2}-\frac{j_{1}}{2}-q_{2}+\beta z_{2}+\alpha z_{3}-z_{1}+1}} \times \frac{\Gamma\left(\frac{\gamma j_{1}}{2}+j_{2}\right) \Gamma\left(\frac{q_{1}}{2}+1\right) \Gamma\left(z_{1}+1\right) \Gamma\left(\gamma z_{2}+z_{3}\right)}{\Gamma\left(\frac{\gamma j_{1}}{2}\right) \Gamma\left(\frac{q_{1}}{2}+1-q_{2}\right) \Gamma\left(z_{1}+1-z_{2}\right) \Gamma\left(\gamma z_{2}\right)}, \\
& \overline{V_{5}}(Y, s)=B_{4} \sum_{n=0}^{\infty} \sum_{t_{1}=0}^{\infty} \sum_{t_{2}=0}^{\infty} \sum_{z_{1}=0}^{\infty} \sum_{z_{2}=0}^{\infty} \sum_{z_{3}}^{\infty} \frac{(Y-2 n-1){ }^{t_{1}}(P r)^{\frac{t_{1}}{2}}\left(-B_{1}\right)^{z_{1}}}{t_{1 !} ! t_{2}: z_{2} ! z_{3} !\left(B_{2}\right)^{2}\left(B_{1}\right)^{z_{2}}} \times \\
& \frac{(-P r)^{z_{2}}(a)^{t_{2}+z_{3}}}{s^{\alpha t_{2}+\frac{\beta t_{1}}{2}-\frac{t_{1}}{2}+\beta z_{2}+\alpha z_{3}-z_{1}+1}} \times \frac{\Gamma\left(\frac{\gamma t_{1}}{2}+t_{2}\right) \Gamma\left(z_{1}+1\right) \Gamma\left(\gamma z_{2}+z_{3}\right)}{\Gamma\left(\frac{\gamma t_{1}}{2}\right) \Gamma\left(z_{1}+1-z_{2}\right) \Gamma\left(\gamma z_{2}\right)}, \\
& \overline{V_{6}}(Y, s)=-B_{4} \sum_{n=0}^{\infty} \sum_{v_{1}=0}^{\infty} \sum_{v_{2}=0}^{\infty} \sum_{z_{1}=0}^{\infty} \sum_{z_{2}=0}^{\infty} \sum_{z_{3}}^{\infty} \frac{(-(Y+2 n+1))^{v_{1}}(P r) \frac{v_{1}}{2}\left(-B_{1}\right)^{z_{1}}}{v_{1} v_{2} ! z_{2} ! z_{3 !}\left(B_{2}\right)^{z_{1}}\left(B_{1}\right)^{z_{2}}} \times \frac{(-P r)^{z_{2}}(a)^{v_{2}+z_{3}}}{s^{\alpha v_{2}+\frac{\beta v_{1}}{2}-\frac{v_{1}}{2}+\beta z_{2}+\alpha z_{3}-z_{1}+1}} \times \\
& \frac{\Gamma\left(\frac{\gamma v_{1}}{2}+v_{2}\right) \Gamma\left(z_{1}+1\right) \Gamma\left(\gamma z_{2}+z_{3}\right)}{\Gamma\left(\frac{\gamma \gamma_{1}}{2}\right) \Gamma\left(z_{1}+1-z_{2}\right) \Gamma\left(\gamma z_{2}\right)},
\end{aligned}
$$

Next, taking the inverse of Equations (34)-(39) Laplace transform, we have 


$$
\begin{aligned}
& V(Y, \tau)=V_{1}(Y, \tau)+V_{2}(Y, \tau)+V_{3}(Y, \tau)+V_{4}(Y, \tau)+V_{5}(Y, \tau)+V_{6}(Y, \tau) \\
& V_{1}(Y, \tau)=-B_{4} \sum_{n=0}^{\infty} \sum_{m=0}^{\infty} \sum_{\delta_{1}=0}^{\infty} \sum_{\delta_{2}=0}^{\infty} \sum_{\omega_{1}=0}^{\infty} \sum_{\omega_{2}=0}^{\infty} \sum_{z_{1}=0}^{\infty} \sum_{z_{2}=0}^{\infty} \sum_{z_{3}=0}^{\infty} \frac{(Y-2 m-1)^{\omega_{1}}\left(-B_{1}\right)^{z_{1}}(P r)^{\frac{\delta_{1}}{2}}}{\delta_{1 !} \delta_{2} ! \omega_{1} ! \omega_{2} ! z_{2} ! z_{3} !\left(B_{1}\right)^{z_{2}-\omega_{2}}\left(B_{2}\right)^{\omega_{2}+z_{1}-\frac{\omega_{1}}{2}}} \times \\
& \frac{(-2 n)^{\delta_{1}}(-P r)^{z_{2}}(a)^{\delta_{2}+z_{3}} \tau^{\alpha \delta_{2}+\frac{\beta \delta_{1}}{2}-\frac{\delta_{1}}{2}-\omega_{2}+\beta z_{2}+\alpha z_{3}-z_{1}}}{\Gamma\left(\alpha \delta_{2}+\frac{\beta \delta_{1}}{2}-\frac{\delta_{1}}{2}-\omega_{2}+\beta z_{2}+\alpha z_{3}-z_{1}+1\right)} \times \frac{\Gamma\left(\frac{\gamma \delta_{1}}{2}+\delta_{2}\right) \Gamma\left(\frac{q_{1}}{2}+1\right) \Gamma\left(z_{1}+1\right) \Gamma\left(\gamma z_{2}+z_{3}\right)}{\Gamma\left(\frac{\gamma \delta_{1}}{2}\right) \Gamma\left(\frac{q_{1}}{2}+1-q_{2}\right) \Gamma\left(z_{1}+1-z_{2}\right) \Gamma\left(\gamma z_{2}\right)}, \\
& V_{2}(Y, \tau)=B_{4} \sum_{n=0}^{\infty} \sum_{m=0}^{\infty} \sum_{\delta_{1}=0}^{\infty} \sum_{\delta_{2}=0}^{\infty} \sum_{q_{1}=0}^{\infty} \sum_{q_{2}=0}^{\infty} \sum_{z_{1}=0}^{\infty} \sum_{z_{2}=0}^{\infty} \sum_{z_{3}=0}^{\infty} \frac{(-(Y+2 m+1))^{q_{1}}\left(-B_{1}\right)^{z_{1}}(\operatorname{Pr})^{\frac{\delta_{1}}{2}}}{\delta_{1 !} \delta_{2} ! q_{1} ! q_{2} ! z_{2} ! z_{3} !\left(B_{1}\right)^{z_{2}-q_{2}}\left(B_{2}\right)^{q_{2}+z_{1}-\frac{q_{1}}{2}}} \times \\
& \frac{(-2 n)^{\delta_{1}}(-P r)^{z_{2}}(a)^{\delta_{2}+z_{3}} \tau^{\alpha \delta_{2}+\frac{\beta \delta_{1}}{2}-\frac{\delta_{1}}{2}-q_{2}+\beta z_{2}+\alpha z_{3}-z_{1}}}{\Gamma\left(\alpha \delta_{2}+\frac{\beta \delta_{1}}{2}-\frac{\delta_{1}}{2}-q_{2}+\beta z_{2}+\alpha z_{3}-z_{1}+1\right)} \times \frac{\Gamma\left(\frac{\gamma \delta_{1}}{2}+\delta_{2}\right) \Gamma\left(\frac{q_{1}}{2}+1\right) \Gamma\left(z_{1}+1\right) \Gamma\left(\gamma z_{2}+z_{3}\right)}{\Gamma\left(\frac{\gamma \delta_{1}}{2}\right) \Gamma\left(\frac{q_{1}}{2}+1-q_{2}\right) \Gamma\left(z_{1}+1-z_{2}\right) \Gamma\left(\gamma z_{2}\right)},
\end{aligned}
$$

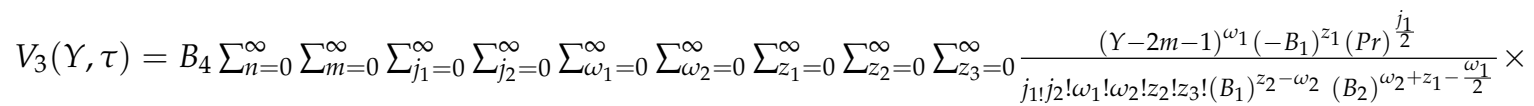

$$
\begin{aligned}
& \frac{(-2 n-2)^{j_{1}}(-P r)^{z_{2}}(a)^{j_{2}+z_{3}} \tau^{\alpha j_{2}+\frac{\beta j_{1}}{2}-\frac{j_{1}}{2}-q_{2}+\beta z_{2}+\alpha z_{3}-z_{1}}}{\Gamma\left(\alpha j_{2}+\frac{\beta j_{1}}{2}-\frac{j_{1}}{2}-q_{2}+\beta z_{2}+\alpha z_{3}-z_{1}+1\right)} \times \frac{\Gamma\left(\frac{\gamma \delta_{1}}{2}+\delta_{2}\right) \Gamma\left(\frac{q_{1}}{2}+1\right) \Gamma\left(z_{1}+1\right) \Gamma\left(\gamma z_{2}+z_{3}\right)}{\Gamma\left(\frac{\gamma \delta_{1}}{2}\right) \Gamma\left(\frac{q_{1}}{2}+1-q_{2}\right) \Gamma\left(z_{1}+1-z_{2}\right) \Gamma\left(\gamma z_{2}\right)}, \\
& V_{4}(Y, \tau)=-B_{4} \sum_{n=0}^{\infty} \sum_{m=0}^{\infty} \sum_{j_{1}=0}^{\infty} \sum_{j_{2}=0}^{\infty} \sum_{q_{1}=0}^{\infty} \sum_{q_{2}=0}^{\infty} \sum_{z_{1}=0}^{\infty} \sum_{z_{2}=0}^{\infty} \sum_{z_{3}=0}^{\infty} \frac{(-(Y+2 m+1))^{q_{1}}\left(-B_{1}\right)^{z_{1}}(P r)^{\frac{j_{1}}{2}}}{j_{1} j ! q_{1} ! q_{2} ! z_{2} ! z_{3} !\left(B_{1}\right)^{z_{2}-q_{2}}\left(B_{2}\right)^{q_{2}+z_{1}-\frac{q_{1}}{2}}} \times \\
& \frac{(-2 n-2)^{j_{1}}(-P r)^{z_{2}}(a)^{j_{2}+z_{3}} \tau^{\alpha j_{2}}+\frac{\beta j_{1}}{2}-\frac{j_{1}}{2}-q_{2}+\beta z_{2}+\alpha z_{3}-z_{1}}{\Gamma\left(\alpha j_{2}+\frac{\beta j_{1}}{2}-\frac{j_{1}}{2}-q 2+\beta z_{2}+\alpha z_{3}-z_{1}+1\right)} \times \frac{\Gamma\left(\frac{\gamma j_{1}}{2}+j_{2}\right) \Gamma\left(\frac{q_{1}}{2}+1\right) \Gamma\left(z_{1}+1\right) \Gamma\left(\gamma z_{2}+z_{3}\right)}{\Gamma\left(\frac{\gamma j_{1}}{2}\right) \Gamma\left(\frac{q_{1}}{2}+1-q_{2}\right) \Gamma\left(z_{1}+1-z_{2}\right) \Gamma\left(\gamma z_{2}\right)}, \\
& V_{6}(Y, \tau)=-B_{4} \sum_{n=0}^{\infty} \sum_{v_{1}=0}^{\infty} \sum_{v_{2}=0}^{\infty} \sum_{z_{1}=0}^{\infty} \sum_{z_{2}=0}^{\infty} \sum_{z_{3}}^{\infty} \frac{(-(Y+2 n+1))^{v_{1}}(P r)^{\frac{v_{1}}{2}}\left(-B_{1}\right)^{z_{1}}}{v_{1 !} v_{2} ! z_{2} ! z_{3 !}\left(B_{2}\right)^{z_{1}}\left(B_{1}\right)^{z_{2}}} \times \frac{(-P r)^{z_{2}}(a)^{v_{2}+z_{3}} \tau^{\alpha v_{2}}+\frac{\beta v_{1}}{2}-\frac{v_{1}}{2}+\beta z_{2}+\alpha z_{3}-z_{1}}{\Gamma\left(\alpha v_{2}+\frac{\beta v_{1}}{2}-\frac{v_{1}}{2}+\beta z_{2}+\alpha z_{3}-z_{1}+1\right)} \times \\
& \frac{\Gamma\left(\frac{\gamma v_{1}}{2}+v_{2}\right) \Gamma\left(z_{1}+1\right) \Gamma\left(\gamma z_{2}+z_{3}\right)}{\Gamma\left(\frac{\gamma v_{1}}{2}\right) \Gamma\left(z_{1}+1-z_{2}\right) \Gamma\left(\gamma z_{2}\right)},
\end{aligned}
$$

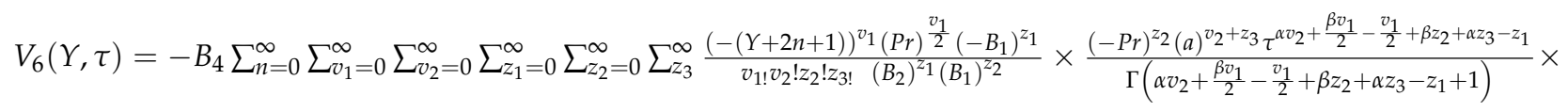

$$
\begin{aligned}
& \frac{\Gamma\left(\frac{\gamma v_{1}}{2}+v_{2}\right) \Gamma\left(z_{1}+1\right) \Gamma\left(\gamma z_{2}+z_{3}\right)}{\Gamma\left(\frac{\gamma v_{1}}{2}\right) \Gamma\left(z_{1}+1-z_{2}\right) \Gamma\left(\gamma z_{2}\right)} .
\end{aligned}
$$

\section{Discussion}

Analytical solutions of heat transfer flow of a Brinkman fluid with a Prabhakar fractional derivative are obtained with the Laplace transform method. In order to see the physical insight of flow parameters, and especially fractional parameters, some graphs are presented in Figures 2-13. Moreover, we have applied a time-fractional operator in a heat transfer problem. We plotted Figures 2 and 3 for the temperature and velocity fluids for different values of fractional parameters by considering small and large values of the time. It is evident from the Figures that for a large time the fluid properties can be enhanced by considering greater values of fractional parameters. However, in Figures 4 and 5, an opposite trend was observed for small values of the time. It is concluded that the fractional solutions show dual behavior for a different time. The fractional parameters can be used to control the thermal and momentum boundary layer, respectively. 


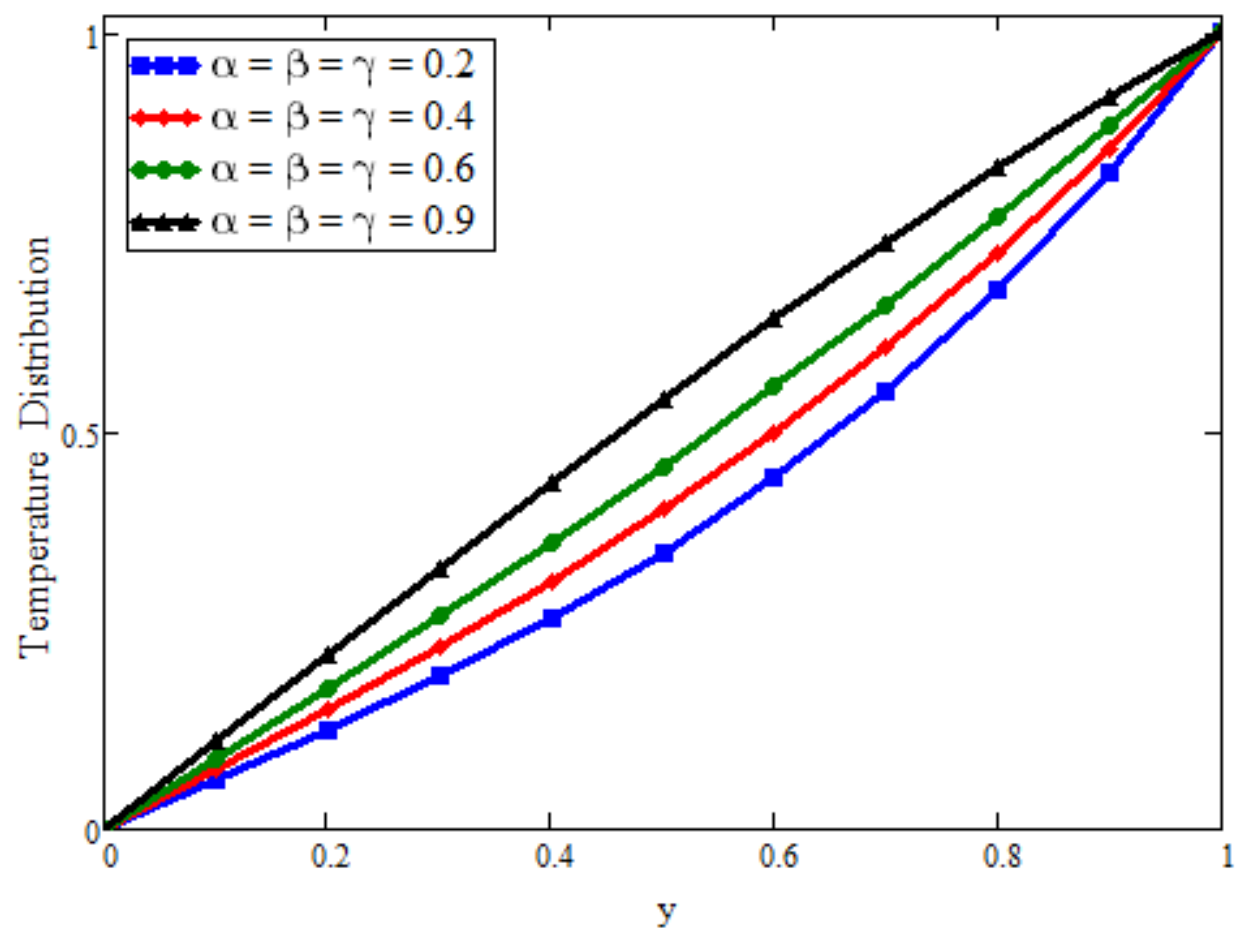

Figure 2. Temperature distribution against y due to fractional parameters $\alpha, \beta, \gamma$ for large time, when $t=3, \operatorname{Pr}=6.2, \phi_{\text {hnf }}=0.02$.

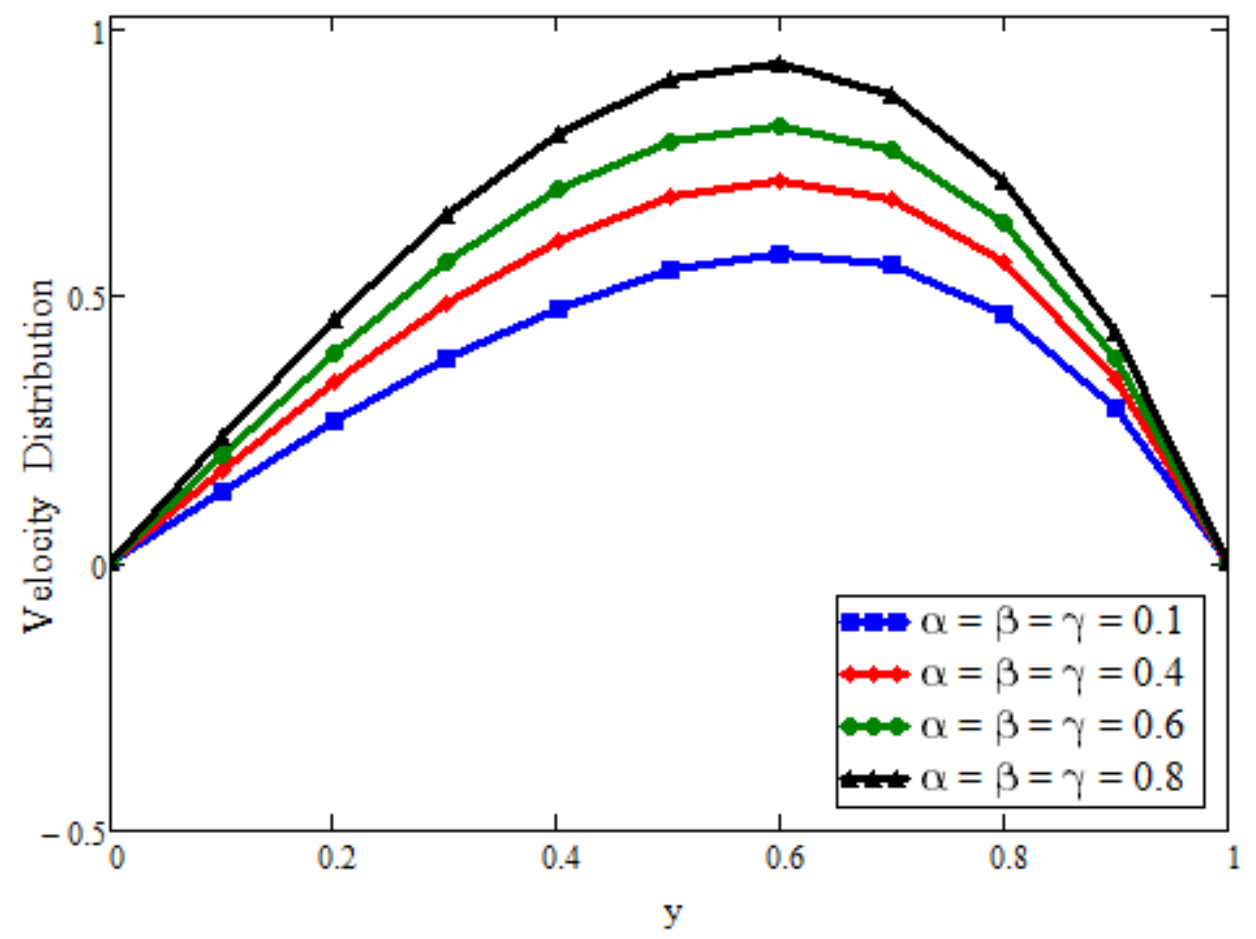

Figure 3. Velocity distribution against y due to for fractional parameters $\alpha, \beta, \gamma$ for large time, when $t=3, \operatorname{Pr}=6.2, \phi_{h n f}=0.04, G r=15, M=0.1$. 


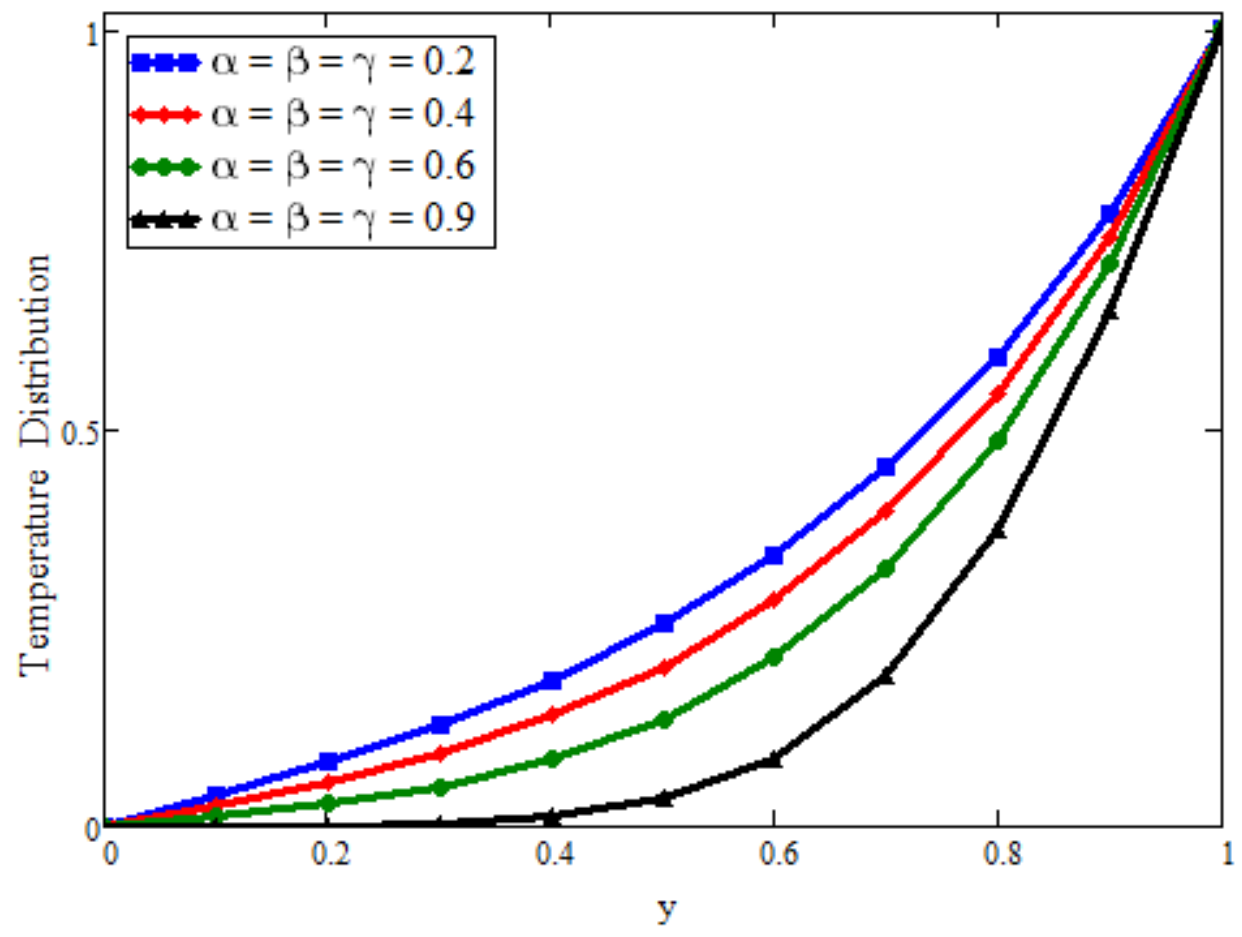

Figure 4. Temperature distribution against y due to fractional parameters $\alpha, \beta, \gamma$ for small time, when $t=0.1, \operatorname{Pr}=6.2, \phi_{\text {hnf }}=0.02$.

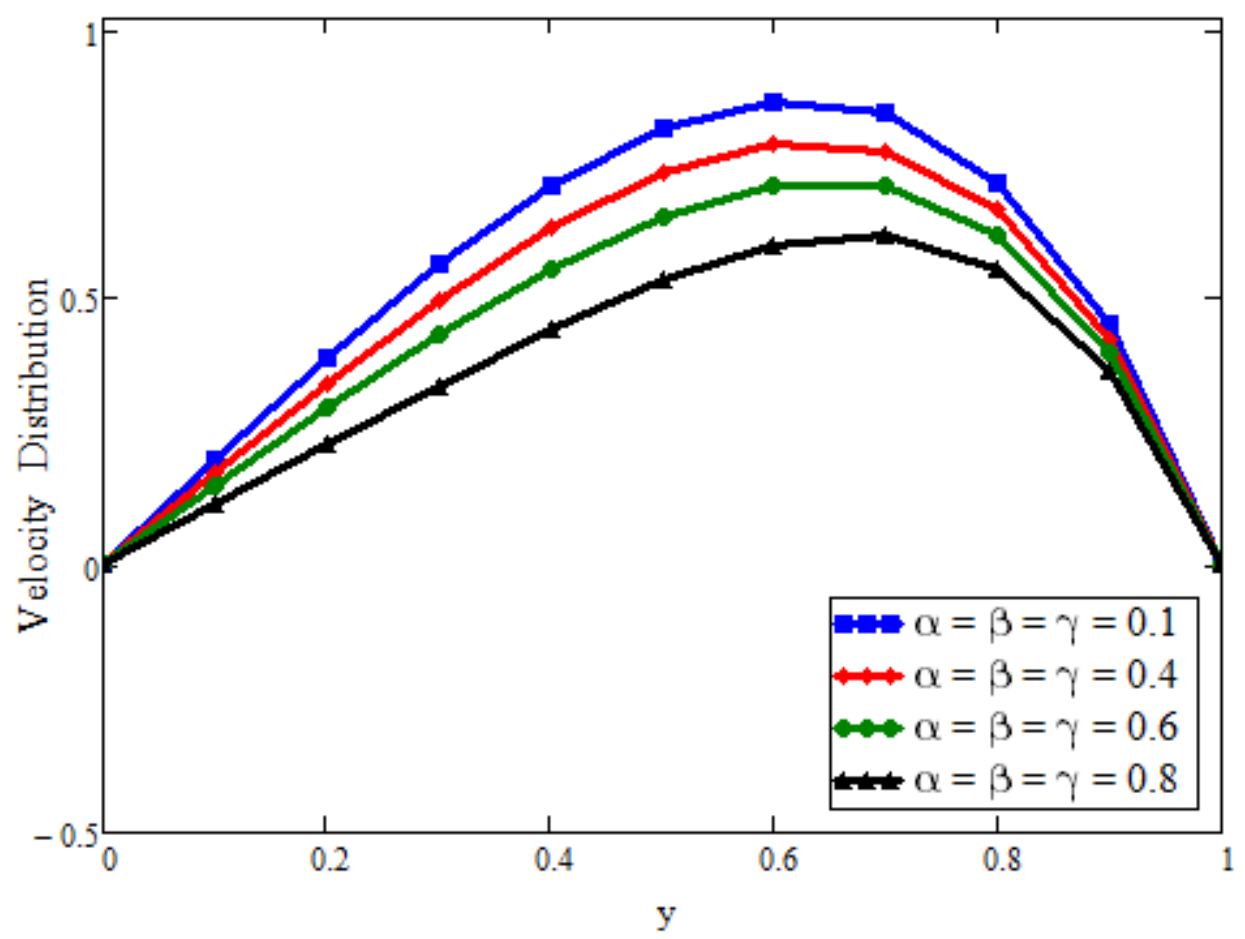

Figure 5. Velocity distribution against y due to fractional parameters $\alpha, \beta, \gamma$ for small time, when $t=0.3, \operatorname{Pr}=6.2, \phi_{\text {hnf }}=0.04, G r=25, M=0.1$. 


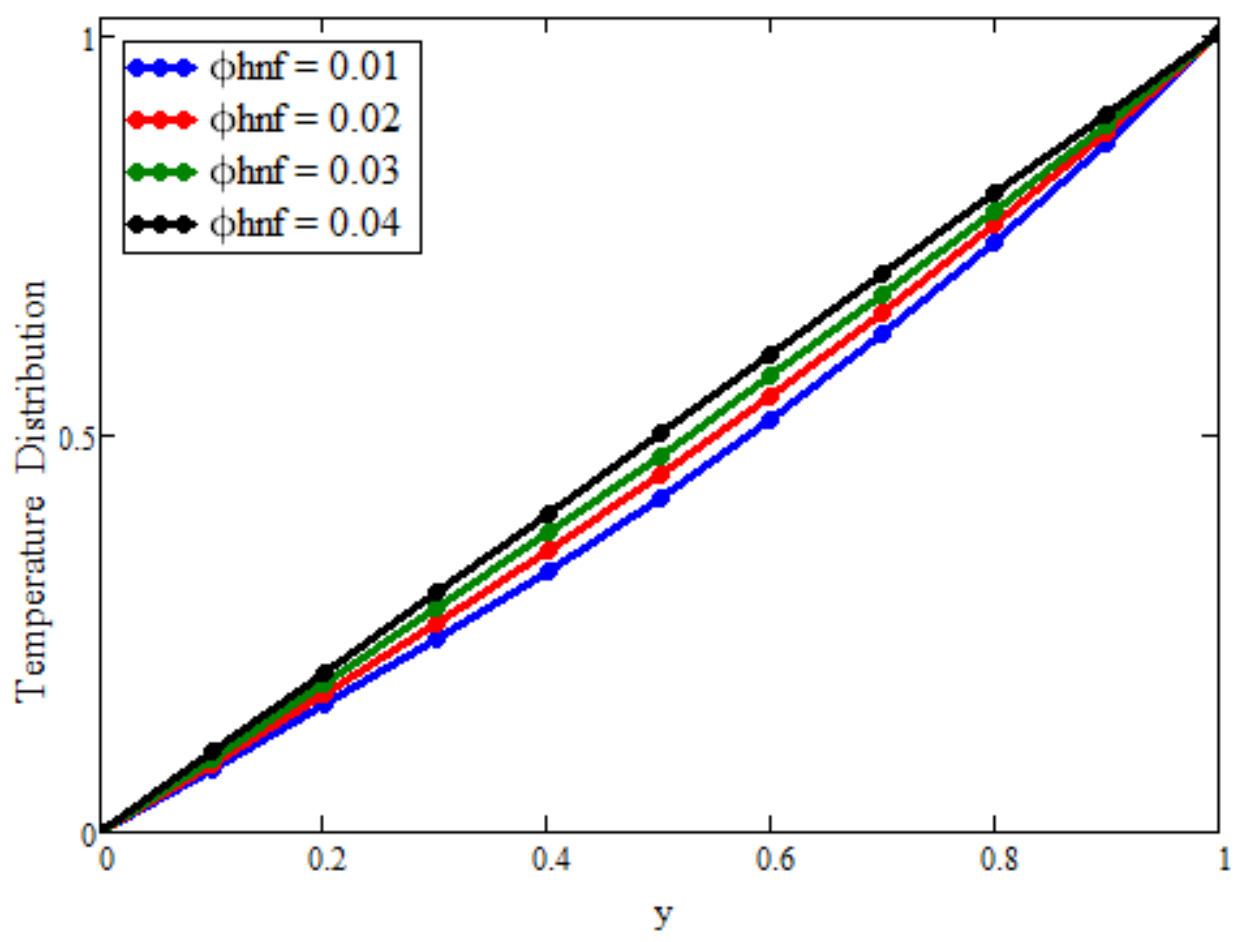

Figure 6. Temperature distribution against y due to $\phi_{h n f}$, when $t=3, \operatorname{Pr}=6.2, \alpha=\beta=$ $\gamma=0.5$

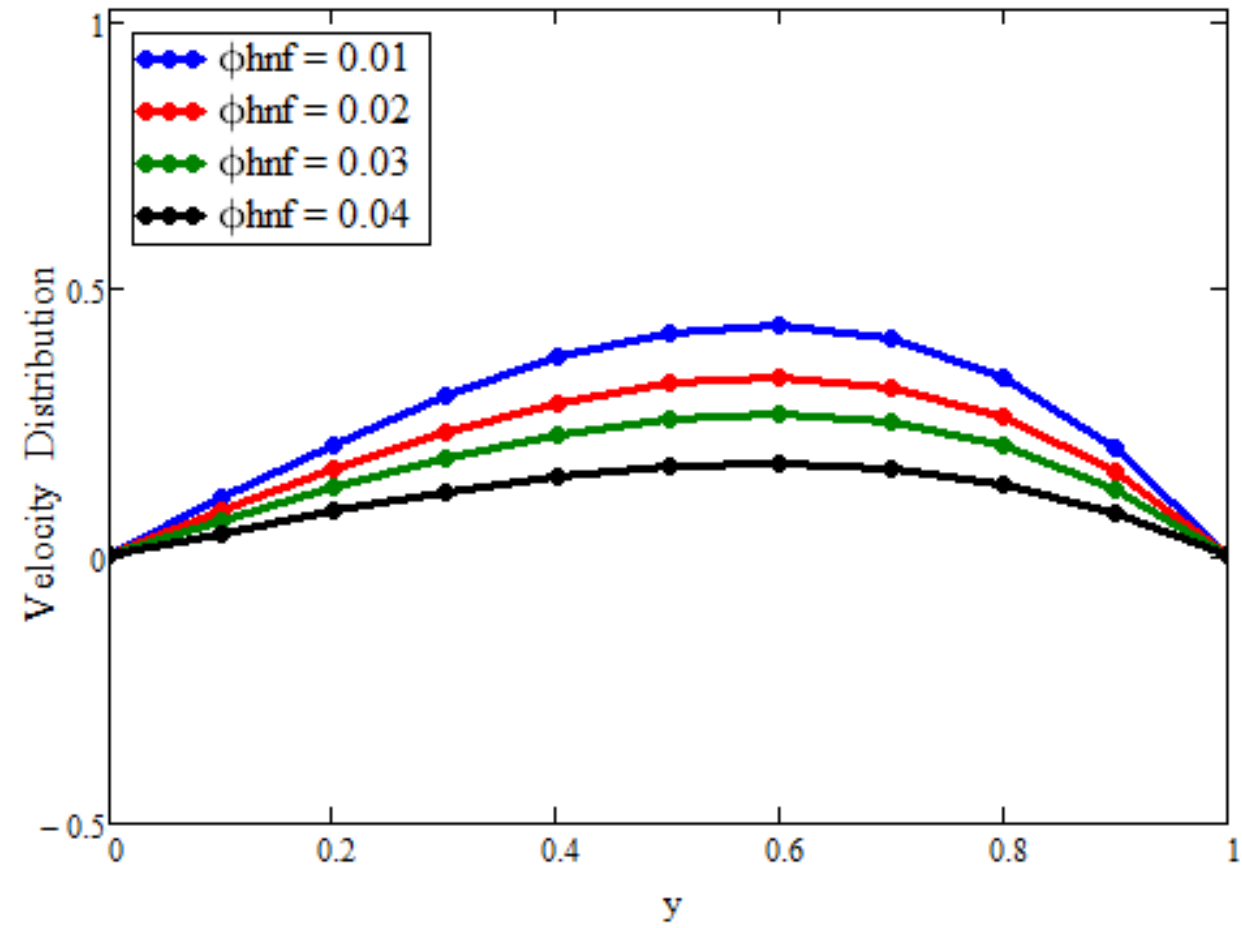

Figure 7. Velocity distribution against y due to $\phi_{h n f}$, when $t=40, \operatorname{Pr}=6.2, G r=15, M=2$, $\alpha=\beta=\gamma=0.5$. 


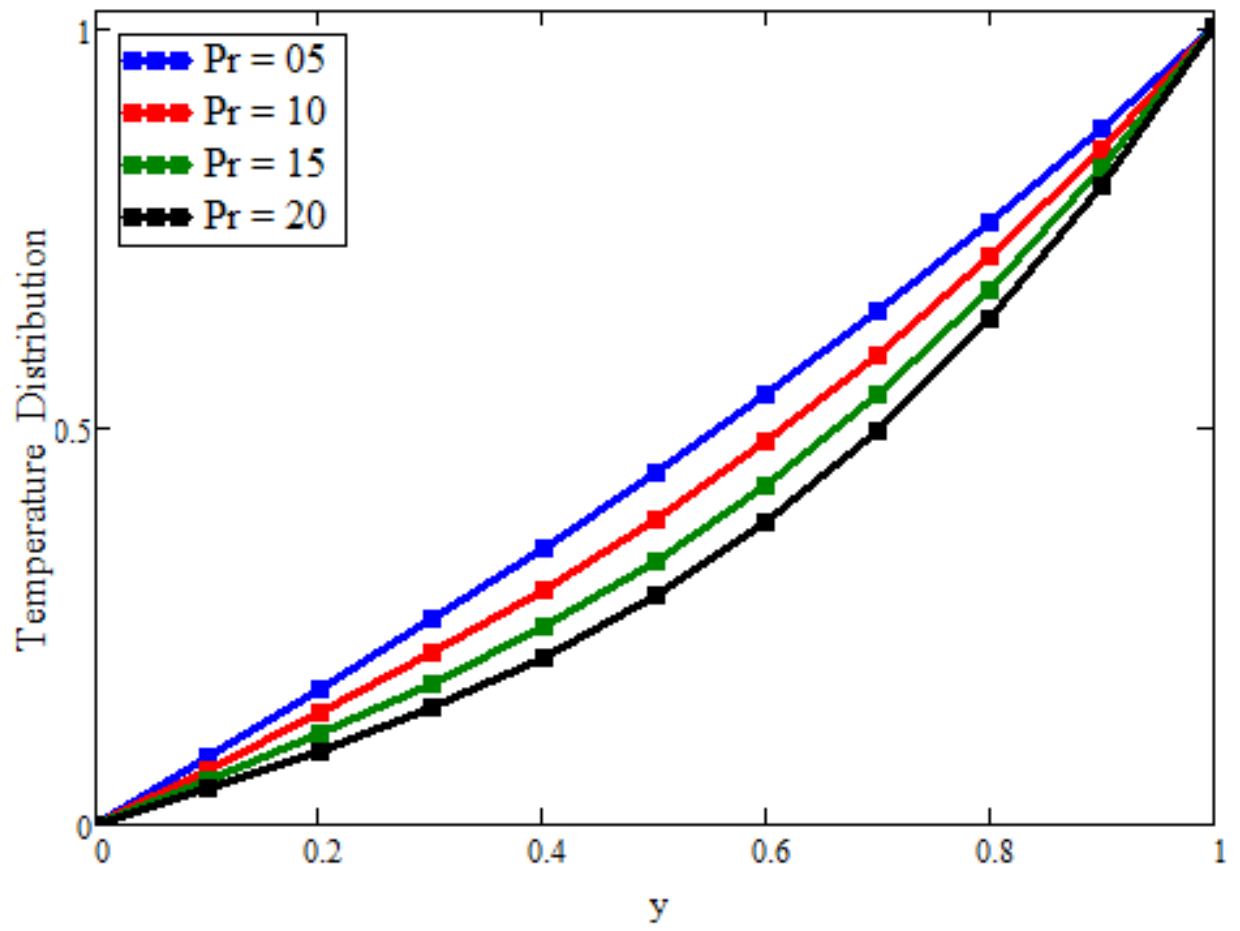

Figure 8. Temperature distribution against y due to $\operatorname{Pr}$, when $t=3, \phi_{\text {hnf }}=0.02, \alpha=\beta=\gamma=0.5$.

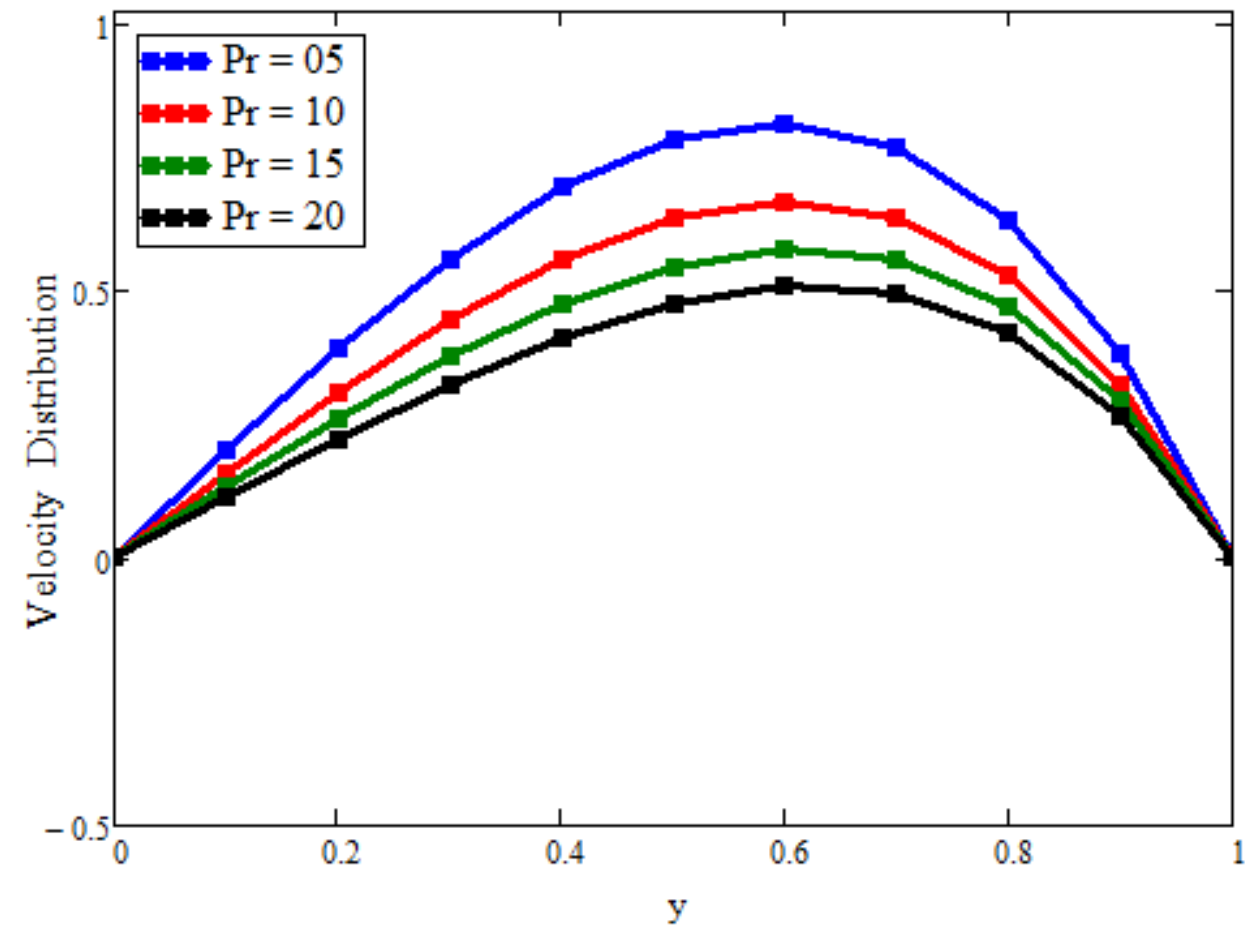

Figure 9. Velocity distribution against y due to $P r$, when $t=3, \phi_{\text {hnf }}=0.04, G r=15, M=0.1$, $\alpha=\beta=\gamma=0.5$. 


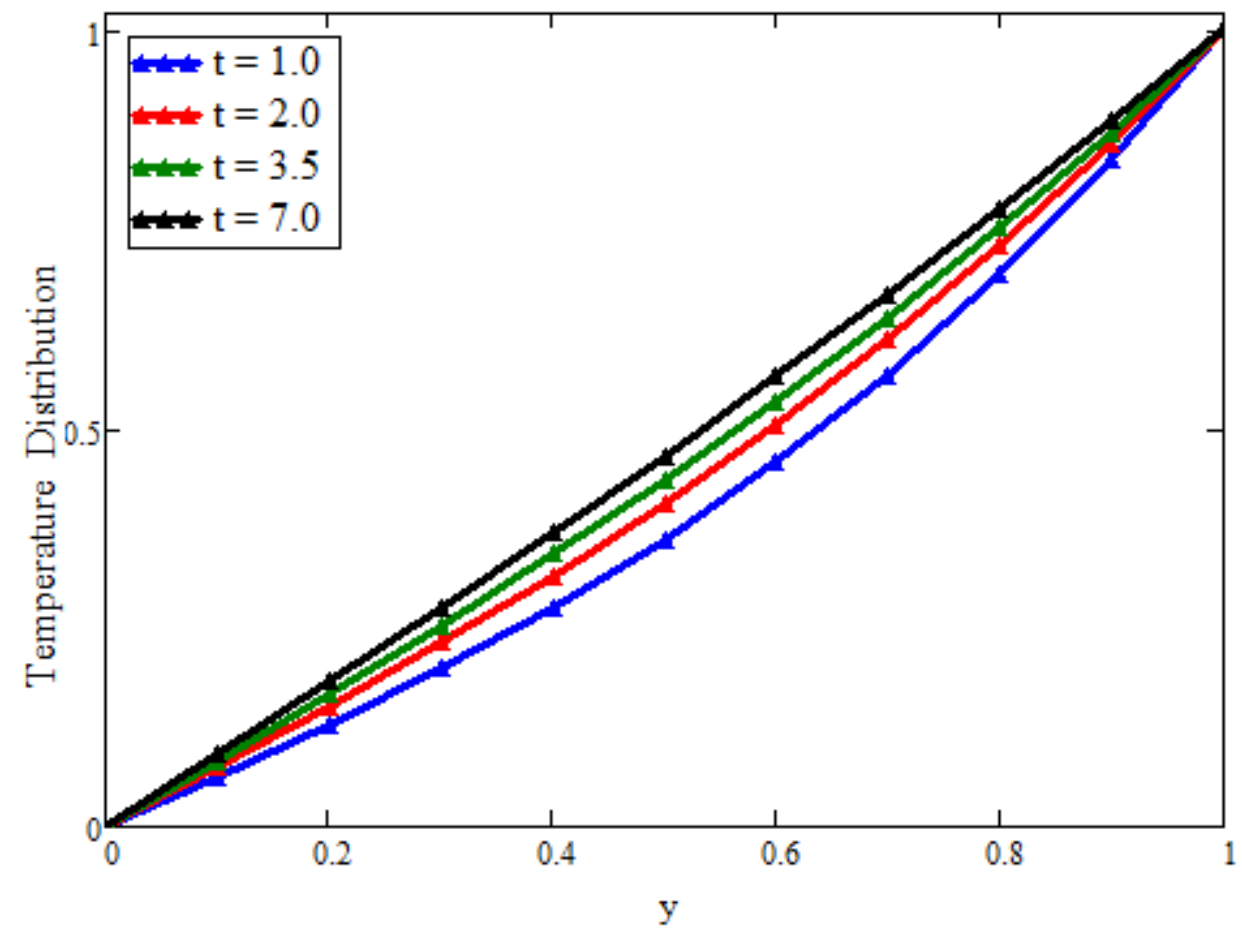

Figure 10. Temperature distribution against y due to $t$, when $\operatorname{Pr}=6.2, \phi_{\text {hnf }}=0.02, \alpha=\beta=\gamma=0.5$.

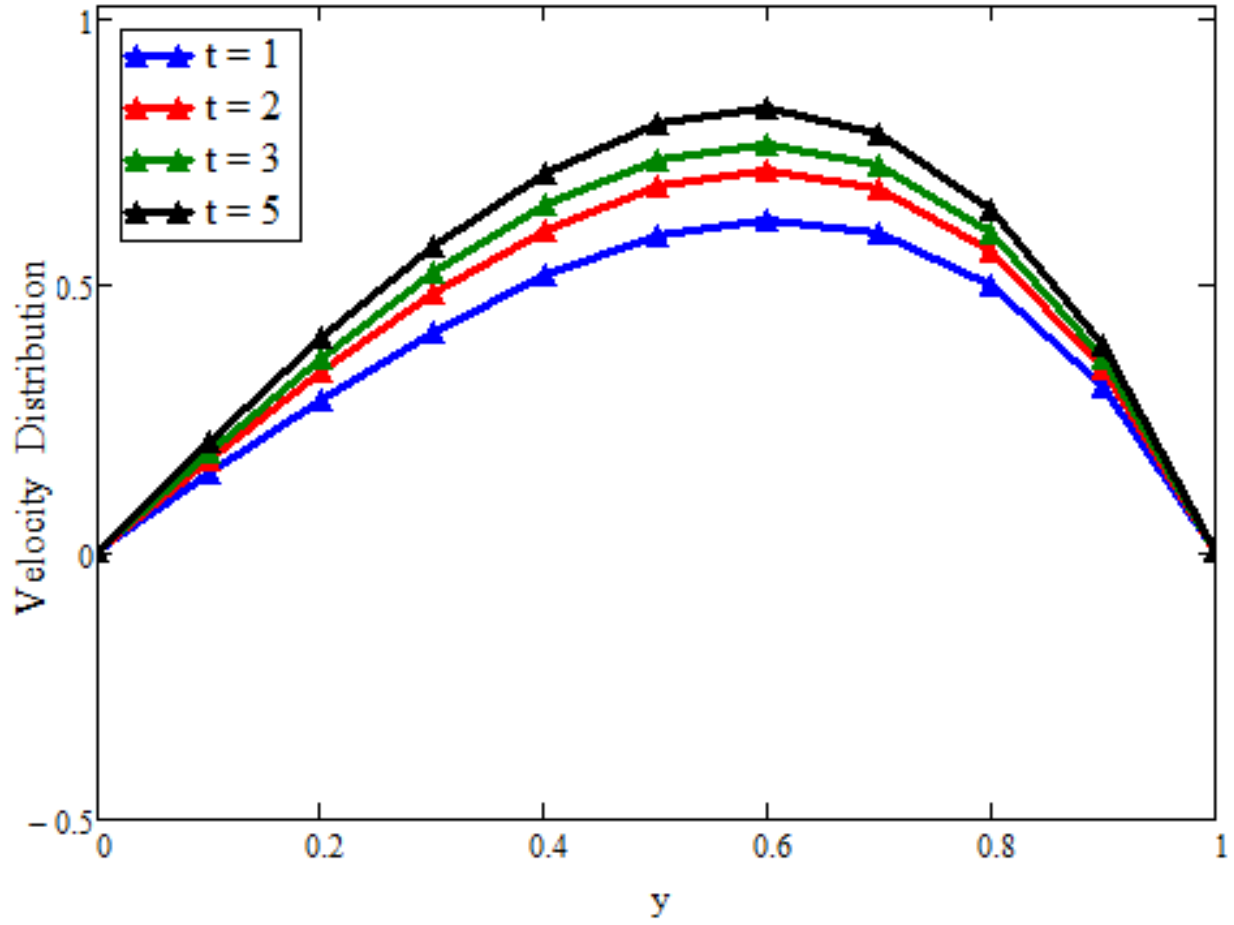

Figure 11. Velocity distribution against y due to $t$, when $\operatorname{Pr}=6.2, \phi_{\text {hnf }}=0.04, G r=15, M=0.1$, $\alpha=\beta=\gamma=0.5$. 


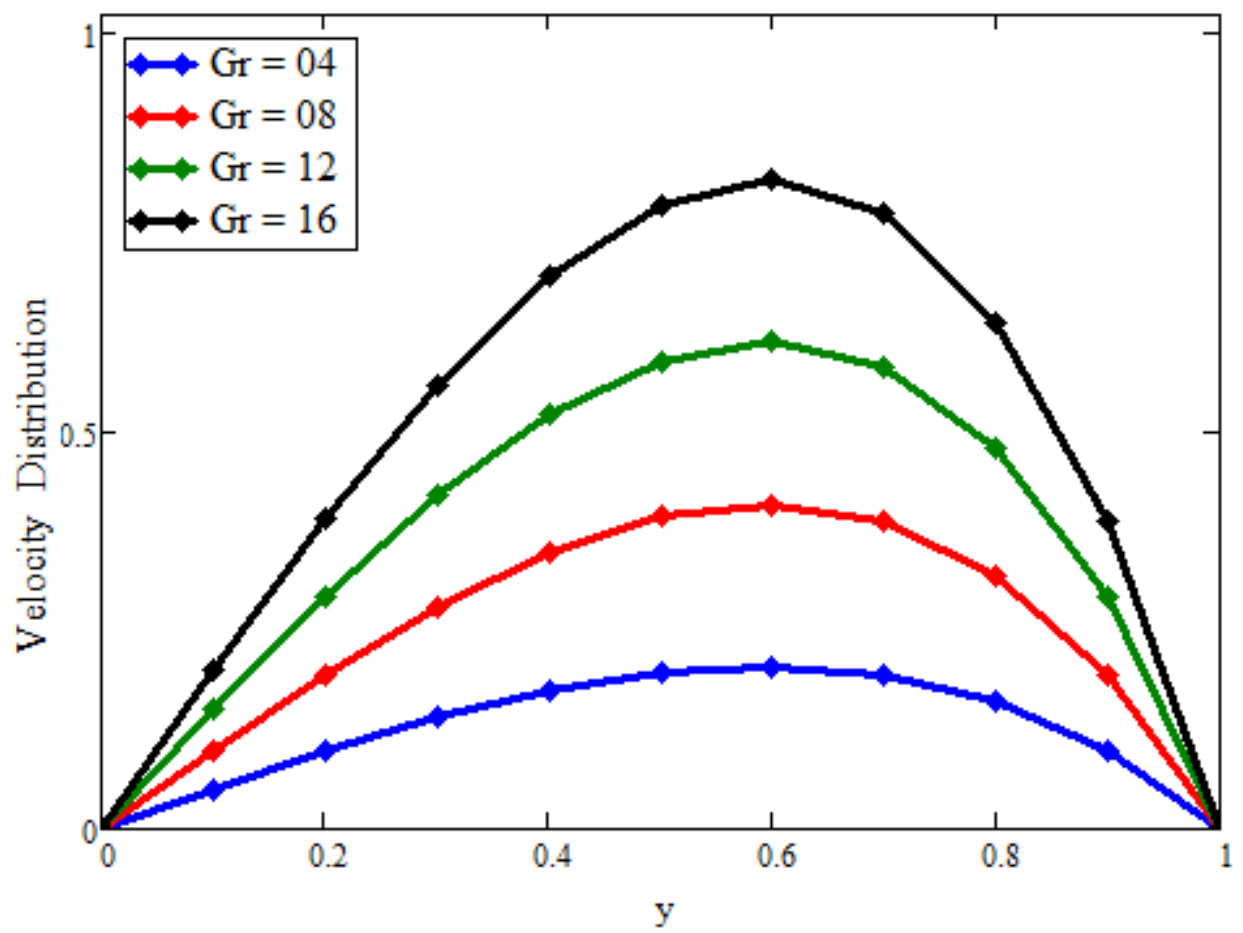

Figure 12. Velocity distribution against y due to $G r$, when $\operatorname{Pr}=6.2, \phi_{\text {hnf }}=0.04, t=3$, $M=0.1, \alpha=\beta=\gamma=0.5$.

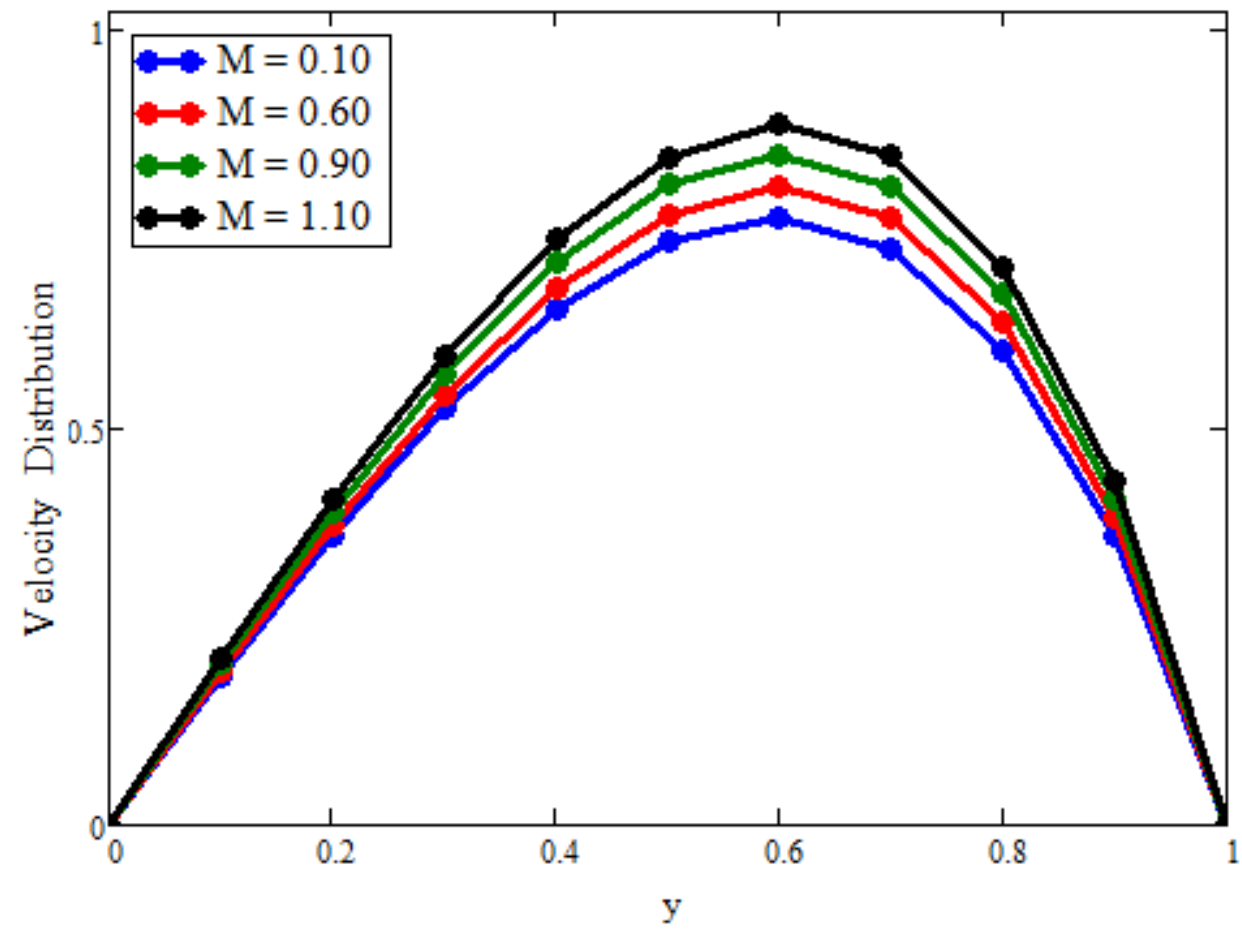

Figure 13. Velocity distribution against y due to $M$, when $\operatorname{Pr}=6.2, \phi_{\text {hnf }}=0.04, t=3$, Gr $=15, \alpha=\beta=\gamma=0.5$.

The influence of $\phi_{h n f}$ volume fraction of hybrid nanoparticles is depicted in Figures 6 and 7. It is found that the temperature can be enhanced for greater values of $\phi_{h n f}$ and velocity depicted declines, respectively. For example, from $\phi_{h n f}=0$, the base fluid is water and has greater velocity, but on increasing $\phi_{h n f}=0.01,0.02,0.04$, decay was observed in velocity. Physically, for large values of $\phi_{h n f}$, the viscous forces dominate and 
finally velocity decreases. As a result, water-based hybrid nanoparticles are denser than pure water.

Temperature decreases when increasing the value of Pr in Figure 8. Physically, the thermal conductivity increasing the values of Pr, making fluid thicker, causes the minimum thickness of the thermal boundary layer. The effect of the velocity profile on Pr is shown in Figure 9. Velocity reduces for bigger values of Pr. Actually, the fluids have bigger viscosity for a large Pr number, and minimum thermal conductivity reduces the velocity due to thicker fluid. In the following, the influence of time to temperature and velocity can be seen in Figures 10 and 11. Time advance causes the increase of the temperature as well as velocity.

Velocity increases as we increase the values of Gr in Figure 12. This is because when Gr is increased, the buoyancy forces become stronger, which results in more convection taking place. Therefore, after that, the velocity increases. Figure 13 shows that the velocity decreases as we rise the values of M. Physically, it can occur that it answers to the drag force, which has an influence on the velocity profile that faces the fluid motion. As a result, velocity decreases.

\section{Conclusions}

This paper deals with the analytical solutions of fractional partial differential equations appearing in heat transport phenomena. The analysis includes base fluid modeled as water together with different nanoparticles. The solutions are obtained via the Laplace transform method, and the following conclusions can be drawn:

a. Dual solutions for both temperature and velocity fields are predicted for different values of fractional parameters describing generalized. Fourier's law (based on Prabhakar's fractional derivative);

b. Fluid property, i.e., temperature, can be enhanced by increasing the concentration level of nanoparticles;

c. Finally, the fractional parameters (which accomplish the heat memory impacts) can control the thermal and momentum boundary layer thickness;

d. The obtained solutions can be beneficial for proper analysis of real data and provide a tool for testing possible approximate solutions where needed.

Author Contributions: M.I.A. Formal analysis, Conceptualization, N.S. Visualization, Validation. M.B.H. original draft: Software, Investigation, W.S. Funding acquisition, Supervision, Project administration, T.M. Resources, Writing-review \& editing. All authors have read and agreed to the published version of the manuscript.

Funding: The research was funded by National Science Center grant number 2017/27/B/ST8/0351.

Institutional Review Board Statement: Not applicable.

Informed Consent Statement: Not applicable.

Data Availability Statement: The data that support the findings of this study are available within the article.

Acknowledgments: The authors extend their appreciation to the Deanship of Scientific Research at King Khalid University, Abha, Saudi Arabia, for funding this work through the general research groups program under grant number GRP/342/42.

Conflicts of Interest: There is no conflict of interest.

\section{References}

1. Bräuer, F.; Trautner, E.; Hasslberger, J.; Cifani, P.; Klein, M. Turbulent Bubble-Laden Channel Flow of Power-Law Fluids: A Direct Numerical Simulation Study. Fluids 2021, 6, 40. [CrossRef]

2. Zheng, Y.; Yang, H.; Mazaheri, H.; Aghaei, A.; Mokhtari, N.; Afrand, M. An investigation on the influence of the shape of the vortex generator on fluid flow and turbulent heat transfer of hybrid nanofluid in a channel. J. Therm. Anal. Calorim. 2021, 143, 1425-1438. [CrossRef] 
3. D'Ippolito, A.; Calomino, F.; Alfonsi, G.; Lauria, A. Flow Resistance in Open Channel Due to Vegetation at Reach Scale: A Review. Water 2021, 13, 116. [CrossRef]

4. Choi, S.U.; Eastman, J.A. Enhancing Thermal Conductivity of Fluids with Nanoparticles (No. ANL/MSD/CP-84938.CONF-951135-29); Argonne National Lab.: Argonne, IL, USA, 1995.

5. Nadeem, S.; Ahmad, S.; Khan, M.N. Mixed convection flow of hybrid nanoparticle along a Riga surface with Thomson and Troian slip condition. J. Therm. Anal. Calorim. 2021, 143, 2099-2109. [CrossRef]

6. Reddy, M.G.; Shehzad, S.A. Molybdenum disulfide and magnesium oxide nanoparticle performance on micropolar CattaneoChristov heat flux model. Appl. Math. Mech. 2021, 42, 541-552. [CrossRef]

7. Wole-Osho, I.; Okonkwo, E.C.; Adun, H.; Kavaz, D.; Abbasoglu, S. An intelligent approach to predicting the effect of nanoparticle mixture ratio, concentration and temperature on thermal conductivity of hybrid nanofluids. J. Therm. Anal. Calorim. 2021, 144, 671-688. [CrossRef]

8. Haque, M.E.; Hossain, M.S.; Ali, H.M. Laminar forced convection heat transfer of nanofluids inside noncircular ducts: A review. Powder Technol. 2020. [CrossRef]

9. Khashi'ie, N.S.; Arifin, N.M.; Pop, I.; Nazar, R. Melting heat transfer in hybrid nanofluid flow along a moving surface. J. Therm. Anal. Calorim. 2020. [CrossRef]

10. Goldanlou, A.S.; Sepehrirad, M.; Papi, M.; Hussein, A.K.; Afrand, M.; Rostami, S. Heat transfer of hybrid nanofluid in a shell and tube heat exchanger equipped with blade-shape turbulators. J. Therm. Anal. Calorim. 2021, 143, 1689-1700. [CrossRef]

11. Montazer, E.; Shafii, M.B.; Salami, E.; Muhamad, M.R.; Yarmand, H.; Gharehkhani, S.; Badarudin, A. Heat transfer in turbulent nanofluids: Separation flow studies and development of novel correlations. Adv. Powder Technol. 2020, 31, 3120-3133. [CrossRef]

12. Benkhedda, M.; Boufendi, T.; Tayebi, T.; Chamkha, A.J. Convective heat transfer performance of hybrid nanofluid in a horizontal pipe considering nanoparticles shapes effect. J. Therm. Anal. Calorim. 2020, 140, 411-425. [CrossRef]

13. Bhattad, A.; Sarkar, J.; Ghosh, P. Hydrothermal performance of different alumina hybrid nanofluid types in plate heat exchanger. J. Therm. Anal. Calorim. 2020, 139, 3777-3787. [CrossRef]

14. Izadi, M. Effects of porous material on transient natural convection heat transfer of nanofluids inside a triangular chamber. Chin. J. Chem. Eng. 2020, 28, 1203-1213. [CrossRef]

15. Waini, I.; Ishak, A.; Groşan, T.; Pop, I. Mixed convection of a hybrid nanofluid flow along a vertical surface embedded in a porous medium. Int. Commun. Heat Mass Transf. 2020, 114, 104565. [CrossRef]

16. Pandya, N.S.; Desai, A.N.; Tiwari, A.K.; Said, Z. Influence of the geometrical parameters and particle concentration levels of hybrid nanofluid on the thermal performance of axial grooved heat pipe. Therm. Sci. Eng. Prog. 2021, 21, 100762. [CrossRef]

17. Babazadeh, H.; Shah, Z.; Ullah, I.; Kumam, P.; Shafee, A. Analysis of hybrid nanofluid behavior within a porous cavity including Lorentz forces and radiation impacts. J. Therm. Anal. Calorim. 2021, 143, 1129-1137. [CrossRef]

18. Asadi, A.; Alarifi, I.M.; Foong, L.K. An experimental study on characterization, stability and dynamic viscosity of CuO-TiO2/water hybrid nanofluid. J. Mol. Liq. 2020, 307, 112987. [CrossRef]

19. Ikram, M.D.; Asjad, M.; Ahmadian, A.; Ferrara, M. A new fractional mathematical model of extraction nanofluids using clay nanoparticles for different based fluids. Math. Methods Appl. Sci. 2020. [CrossRef]

20. Huminic, G.; Huminic, A. Entropy generation of nanofluid and hybrid nanofluid flow in thermal systems: A review. J. Mol. Liq. 2020, 302, 112533. [CrossRef]

21. Nadeem, S.; Abbas, N.; Malik, M.Y. Inspection of hybrid based nanofluid flow over a curved surface. Comput. Methods Programs Biomed. 2020, 189, 105193. [CrossRef]

22. Baleanu, D.; Diethelm, K.; Scalas, E.; Trujillo, J.J. Fractional Calculus: Models and Numerical Methods; World Scientific: London, UK, 2012; Volume 3.

23. Ortigueira, M.D.; Machado, J.T. Fractional derivatives: The perspective of system theory. Mathematics 2019, 7, 150. [CrossRef]

24. Sene, N. Second-grade fluid model with Caputo-Liouville generalised fractional derivative. Chaos Solitons Fractals 2020, 133, 109631. [CrossRef]

25. Mozafarifard, M.; Toghraie, D. Time-fractional subdiffusion model for thin metal films under femtosecond laser pulses based on Caputo fractional derivative to examine anomalous diffusion process. Int. J. Heat Mass Transf. 2020, 153, 119592. [CrossRef]

26. Thabet, S.T.; Abdo, M.S.; Shah, K.; Abdeljawad, T. Study of transmission dynamics of COVID-19 mathematical model under ABC fractional order derivative. Results Phys. 2020, 19, 103507. [CrossRef]

27. Prabhakar, T.R. A Singular Integral Equation with a Generalized Mittag Leffler Function in the Kernel. 1971. Available online: https: / / www.semanticscholar.org/paper / A-SINGULAR-INTEGRAL-EQUATION-WITH-A-GENERALIZED-IN-Prabhakar / cf26590a6a758228228045275591231430db614f (accessed on 2 July 2021).

28. Yang, X.J. General Fractional Derivatives: Theory, Methods and Applications; CRC Press: Boca Raton, FL, USA, 2019.

29. Garra, R.; Gorenflo, R.; Polito, F.; Tomovski, Ž. Hilfer-Prabhakar derivatives and some applications. Appl. Math. Comput. 2014, 242, 576-589. [CrossRef]

30. Fernandez, A.; Baleanu, D. Classes of operators in fractional calculus: A case study. Math. Methods Appl. Sci. 2021. [CrossRef]

31. Giusti, A.; Colombaro, I. Prabhakar-like fractional viscoelasticity. Commun. Nonlinear Sci. Numer. Simul. 2018, 56, 138-143. [CrossRef]

32. Elnaqeeb, T.; Shah, N.A.; Mirza, I.A. Natural convection flows of carbon nanotubes nanofluids with Prabhakar-like thermal transport. Math. Methods Appl. Sci. 2020. [CrossRef] 
33. Shah, N.A.; Fetecau, C.; Vieru, D. Natural convection flows of Prabhakar-like fractional Maxwell fluids with generalized thermal transport. J. Therm. Anal. Calorim. 2021, 143, 2245-2258. [CrossRef]

34. Akgül, A.; Siddique, I. Novel applications of the magnetohydrodynamics couple stress fluid flows between two plates with fractal-fractional derivatives. Numer. Methods Part. Differ. Equ. 2021, 37, 2178-2189. [CrossRef]

35. Wang, F.; Asjad, M.I.; Zahid, M.; Iqbal, A.; Ahmad, H.; Alsulami, M.D. Unsteady thermal transport flow of Casson nanofluids with generalized Mittag-Leffler kernel of Prabhakar's type. J. Mater. Res. Technol. 2021. [CrossRef]

36. Tashtoush, B.; Magableh, A. Magnetic field effect on heat transfer and fluid flow characteristics of blood flow in multi-stenosis arteries. Heat Mass Transf. 2008, 44, 297-304. [CrossRef]

37. Saqib, M.; Shafie, S.; Khan, I.; Chu, Y.M.; Nisar, K.S. Symmetric MHD channel flow of nonlocal fractional model of BTF containing hybrid nanoparticles. Symmetry 2020, 12, 663. [CrossRef]

38. Polito, F.; Tomovski, Z. Some properties of Prabhakar-type fractional calculus operators. Fract. Differ. Calc. 2016, 6, 73-94. [CrossRef] 